\title{
Unconditional energy dissipation and error estimates of the SAV Fourier spectral method for nonlinear fractional generalized wave equation $^{\star}$
}

\author{
Nan Wang ${ }^{a}$, Meng Li ${ }^{a, *}$, Chengming Huang ${ }^{b, c}$ \\ ${ }^{a}$ School of Mathematics and Statistics, Zhengzhou University, Zhengzhou 450001, China \\ ${ }^{b}$ School of Mathematics and Statistics, Huazhong University of Science and Technology, Wuhan 430074, China \\ ${ }^{c}$ Hubei Key Laboratory of Engineering Modeling and Scientific Computing, Huazhong University of Science and \\ Technology, Wuhan 430074, China
}

\begin{abstract}
In this paper, we consider a second-order scalar auxiliary variable (SAV) Fourier spectral method to solve the nonlinear fractional generalized wave equation. Unconditional energy conservation or dissipation properties of the fully discrete scheme are first established. Next, we utilize the temporal-spatial error splitting argument to obtain unconditional optimal error estimate of the fully discrete scheme, which overcomes time-step restrictions caused by strongly nonlinear system, or the restrictions that the nonlinear term needs to satisfy the assumption of global Lipschitz condition in all previous works for fractional undamped or damped wave equations. Finally, some numerical experiments are presented to confirm our theoretical analysis.
\end{abstract}

Keywords: Fractional generalized wave equation, Fourier spectral method, Scalar auxiliary variable (SAV), Unconditional error estimate, Energy dissipation

\section{Introduction}

In this paper, we consider the following two-dimensional nonlinear space-fractional generalized wave equation (FGWE)

$$
\begin{aligned}
& u_{t t}+\kappa(-\Delta)^{\frac{\alpha}{2}} u+\gamma_{1}(-\Delta)^{\frac{\alpha}{2}} u_{t}+\gamma_{2} u_{t}+F^{\prime}(u)=0, \quad \mathbf{x} \in \Omega, 0<t \leq T, \\
& u(\mathbf{x}, 0)=\phi_{0}(\mathbf{x}), u_{t}(\mathbf{x}, 0)=\varphi_{0}(\mathbf{x}), \mathbf{x} \in \Omega
\end{aligned}
$$

^This work was supported in part by NSF of China $(12001499,11771163,11801527,12011530058)$, China Postdoctoral Science Foundation (2019M662506,2018M632791).

${ }^{*}$ Corresponding author (Meng Li).

Email addresses: nan_wang1991@163.com (N. Wang), limeng@zzu .edu.cn (M. Li*), chengming huang@hotmail. com (C. Huang). 
where the parameters $\kappa>0,1<\alpha \leq 2, \mathbf{x}=\left(x_{1}, x_{2}\right)$ and $\gamma_{1} \geq 0, \gamma_{2} \geq 0$ are coefficients of damping terms. $F(u)$ is nonlinear and nonnegative, and $F^{\prime}(u)$ satisfies local Lipschitz condition. The fractional Laplacian $(-\Delta)^{\alpha}$ is defined by

$$
(-\Delta)^{\alpha} u=\sum_{s, l \in Z}\left(s^{2}+l^{2}\right)^{\alpha} \hat{u}_{s l} e^{i s x_{1}+i l x_{2}}
$$

For $u \in L_{p e r}^{2}(\Omega), u=\sum_{s, l \in \mathbb{Z}} \hat{u}_{s l} e^{i s x_{1}+i l x_{2}}$, where $i^{2}=-1$ and the Fourier coefficients $\hat{u}_{s l}$ are given by

$$
\hat{u}_{s l}=\left(u, e^{i s x_{1}+i l x_{2}}\right)=\frac{1}{|\Omega|} \int_{\Omega} u e^{i s x_{1}+i l x_{2}} d \mathbf{x}
$$

In addition, (1.1)-(1.2) possess the following energy function

$$
H(t)=\int_{\Omega} \frac{1}{2}\left|u_{t}\right|^{2}+\frac{\kappa}{2}\left|(-\Delta)^{\frac{\alpha}{4}} u\right|^{2}+F(u) d \mathbf{x},
$$

and it holds that

$$
\frac{d H(t)}{d t}=-\int_{\Omega}\left(\gamma_{1}\left|(-\Delta)^{\frac{\alpha}{2}} u_{t}\right|^{2}+\gamma_{2}\left|u_{t}\right|^{2}\right) d \mathbf{x}, \quad t \in(0, T] .
$$

The conventional damped $\left(\gamma_{1}=0, \gamma_{2}>0\right)$ or undamped $\left(\gamma_{1}=\gamma_{2}=0\right)$ wave equations are extensively described in anomalous diffusion, hydrology and so on [1-3]. If $F^{\prime}(u)=\sin u,(1.1)$ deduces to the damped sine-Gordon equation, and when $F^{\prime}(u)=u^{3}-1$, (1.1) becomes the damped Klein-Gordon equation. The FGWEs as the generalization of conventional wave equations are widely applied in science and engineering to well describe the long-range interaction, such as the interaction of solitons in a collisionless plasma and the presence of the phenomenon of nonlinear supratransmission of energy [4]. A increasing number of mathematical and numerical methods have been developed for the FGWE. Along the mathematical front, Shomberg [5] proved the wellposedness of the FGWE and also derived the energy dissipation-preserving structure. In general, the analytical solution of the FGWE is difficult to obtain and we have to construct numerical methods.

Along the numerical front, numerous efficient numerical methods for the fractional damped $\left(\gamma_{1}=0, \gamma_{2}>0\right)$ or undamped $\left(\gamma_{1}=\gamma_{2}=0\right)$ wave equation were proposed in recent years. Liu et al. [6] studied a class of unconditionally stable difference schemes based on the Padé approximation for the Riesz space-fractional telegraph equation. Ran and Zhang [7] derived a compact difference scheme with accuracy of fourth-order in space and second-order in time for fractional damped wave equation. Plenty of numerical works in [8-16] have shown that energy dissipative (or conservative) numerical methods have obvious superiority over common numerical methods in long time simulation and thus developing energy dissipation-preserving methods for the fractional wave equation has attracted more and more researchers' attention. For the fractional undamped wave equation, Xin et al. [17] proposed a conservative difference scheme for Riesz space-fractional sine-Gordon equation. Fu et al. [18] derived an explicit structure-preserving algorithm by considering Hamiltonian system for fractional wave equation. For the fractional damped wave equation, Macías-Díaz et al. [19-22] proposed a series of efficient structure-preserving finite difference methods to study the fractional sine-Gordon equation with Riesz fractional derivative. 
Xie et al. [23] proposed a dissipation-preserving fourth-order difference method for fractional damped wave equation. However, we noticed that all existing numerical methods were devoted to investigate the nonlinear fractional wave equation $\left(\gamma_{1}=0, \gamma_{2} \geq 0\right)$ and there is a little attention for the fractional generalized wave equation $\left(\gamma_{1}>0, \gamma_{2}>0\right)$. Recently, Xie et al. [24] developed a dissipation-preserving fourth-order difference method for the nonlinear fractional generalized wave equations with Riesz fractional derivative in two dimensions, and this is the first work to prove unconditional stability and convergence of the fully discrete scheme. But unfortunately, the above scheme is fully implicit, which needs to solve the nonlinear system by a iterative algorithm at each time step, and increases the computational cost. At the same time, the unconditional convergence analysis is under the assumption that $F^{\prime}(u)$ must satisfy global Lipschitz condition, which means that $\left|F^{\prime}(x)-F^{\prime}(y)\right| \leq L|x-y|, x, y \in R$, where $L$ is a positive constant independent of $x$ and $y$. However, this assumption limits the range of applicability. In fact, nonlinear terms in a very large of nonlinear wave equations are the local Lipschitz continuous, i.e., for any $x, y \in \Omega$, $\left|F^{\prime}(x)-F^{\prime}(y)\right| \leq L_{\Omega}|x-y|$, where $L_{\Omega}>0$ is a Lipschitz constant dependent on $\Omega$. For example, if $F^{\prime}(u)=u^{2}$, the nonlinear term satisfies the local Lipschitz condition. These two reasons motivate us to construct a linearly implicit scheme to reduce computational cost and use some other analytical techniques to remove the restriction of the global Lipschitz condition.

There are many energy dissipative schemes in [25-28] for classical nonlinear wave equations. In particular, Jiang et al. [29] extended invariant energy quadratization (IEQ) approach (proposed in [26]), and established a linearly implicit energy-preserving scheme for sine-Gordon equation. Very recently, to overcome introducing an auxiliary function by the IEQ approach, Shen and Xu [30, 31] proposed a new efficient linearly implicit scheme which is called the scalar auxiliary variable (SAV) approach to describe energy dissipative physical systems without the Lipschitz assumption. Moreover, the SAV approach results in a linear system at each time step and it is easy to implement the scheme. Next, Li and Shen [32] gave rigorous error estimate for the stabilized SAV Fourier spectral for the phase field crystal equation. The SAV approach is being studied extensively for other partial differential equations, see Refs. [33, 34] and references therein. Following the superiority of SAV approach, we develop the fully discrete SAV scheme for the FGWE (1.1)-(1.2). In addition, the convergence analysis plays an important role in numerical methods. Most previous works need the requirement of global Lipschitz condition and thus it is necessary to study the convergence analysis under weaker condition. Similar to the technique in [35], Wang et al. [36] recently provided a rigorous convergence analysis for fractional damped wave equation under the condition $\tau^{2} N \leq c$ caused by the inverse inequality as usual, where nonlinear term is under the weaker assumption.The similar time-spatial stepsize restriction often appeared in general nonlinear parabolic equations, and in order to get the unconditional error estimate without the time stepsize restrictions, the temporal-spatial error splitting argument was presented to get unconditional optimal error estimate for parabolic equations [37-39]. Inspired by the technique, Zeng et al. [40] proposed Fourier spectral method for nonlinear fractional reaction-diffusion equation and used the temporal-spatial error splitting argument to overcome the time stepsize restriction in [35]. However, using the temporal-spatial error splitting argument to obtain the unconditional error estimate for the FGWE, has not been studied. In this work, we extend SAV Fourier spectral method for the FGWE, which the resulting system can be used FFT solver. Furthermore, the temporalspatial error splitting argument is adopted to study the unconditional convergence analysis of the 
fully discrete scheme.

The main contribution of this paper is to develop the SAV Fourier-spectral method for the FGWE in two dimensions, which can maintain energy dissipation and reach high error accuracy. The unconditional energy dissipation is proved in detail. We use temporal-spatial error splitting argument to obtain the unconditional optimal error estimate without the global Lipschitz assumption. We obtain that our scheme is convergent with second order accuracy in time and spectral accuracy in space. Numerical experiments are provided to confirm the theoretical results and validate the efficiency of our algorithms.

The rest of paper is organized as follows. In Section 2, we recall some technical lemmas and notations. In Section 3, we present the SAV spectral method for FGWE and main results. Unconditional energy dissipation and optimal error estimate results of the fully discrete scheme are shown. We get time-discrete system based on SAV approach and give error estimate as well as unconditional energy dissipation for time-discrete system in Section 4. The boundedness of numerical solutions in $L^{\infty}$ norm are proved unconditionally in Section 5. In section 6, the optimal convergence analysis of the SAV spectral scheme based on the temporal error estimate and the spatial error estimate is completed. The numerical experiments are performed to confirm the correctness of theoretical analysis in Section 7. Some conclusions are drawn in Section 8.

\section{Preliminaries}

In this section, we first recall some essential notations and lemmas. Denote $\Omega$ be a finite domain with $\Omega=I_{x} \times I_{y}=(a, b) \times(c, d)$. Let $C_{p e r}^{\infty}(\Omega)$ be the set of all restrictions onto $\Omega$ of all complex-values, $2 \pi$-periodic, $C^{\infty}$ function on $\mathbb{R}^{2}$. For a nonnegative real number $r$, let $H_{p e r}^{r}(\Omega)$ be the closure of $C_{p e r}^{\infty}(\Omega)$ with the semi-norm $|\cdot|_{r}$ and norm $\|\cdot\|_{r}$ defined by

$$
|u|_{r}^{2}=\sum_{s, l \in Z} \hat{u}_{s, l}^{2}\left(s^{2}+l^{2}\right)^{r}, \quad\|u\|_{r}^{2}=\sum_{s, l \in Z} \hat{u}_{s, l}^{2}\left(1+s^{2}+l^{2}\right)^{r} .
$$

Lemma 2.1. ([41]). Let $\xi, r \geq 0$, then for any $u, v \in H_{p e r}^{\xi+r}(\Omega)$, it hold that

$$
\left((-\Delta)^{\xi+r} u, v\right)=\left((-\Delta)^{\xi} u,(-\Delta)^{r} v\right) .
$$

Lemma 2.2. ([40, 41]). If $\xi<r$ and $u, v \in H_{p e r}^{2 \xi}(\Omega)$ for all $\xi>0$, then

$$
\left.\left\|(-\Delta)^{\xi}(u v)\right\|^{2} \leq C_{\xi}\left[\|v\|_{\infty}^{2}\left\|(-\Delta)^{\xi} u\right\|^{2}\right]+\left\|(-\Delta)^{\xi} v\right\|^{2}\|u\|_{\infty}^{2}\right]
$$

where $C_{\xi}=\max \left\{1,2^{2 \xi-1}\right\}$.

Lemma 2.3. ([40] 42]]). Let $\Omega$ be a domain in $\mathbb{R}^{n}$ satisfying the cone condition. If $m p>n$, let $p \leq q \leq \infty$; if $m p<n$, let $p \leq q \leq q^{*}=n p /(n-m p)$. Then there exists a positive constant $C_{1}$ depending on $m, n, p, q$, and the dimensions of $\Omega$ such that for all $u \in W^{m, p}(\Omega)$,

$$
\|u\|_{q} \leq C_{1}\|u\|_{m, p}^{\varepsilon}\|u\|_{p}^{1-\varepsilon}
$$

where $\varepsilon=(n / m p)-(n / m q)$. 
Lemma 2.4. ([40, 43]]). For any $u \in X_{N}$, there existing a positive constant $C_{2}$ independent of $N$ and the following inverse inequality holds,

$$
\|u\|_{\infty} \leq C_{2} N\|u\|
$$

Lemma 2.5. ([41]]). Suppose that $u \in H_{\text {per }}^{r}(\Omega)$. Then the following estimate holds for all $0 \leq \xi \leq r$,

$$
\left\|u-P_{N} u\right\|_{\xi} \leq C_{3} N^{\xi-r}\|u\|_{r}
$$

where $C_{3}$ is a positive constant not depending on $N$.

\section{The SAV Fourier spectral method for two-dimensional FGWE and main results}

In this section, a linealy implicit fully discrete scheme is constructed, which is based on the SAV approach in time and Fourier spectral method in space. Moreover, we give theoretical analysis of the linear system, including the unconditional energy dissipation and the corresponding error estimate.

\subsection{The stabilized SAV approach}

The equation (1.1)-(1.2), by introducing a scalar variable $r(t)=\sqrt{E(u)}$, can be transformed into the following system

$$
\begin{aligned}
& u_{t}=v, \\
& v_{t}+\kappa(-\Delta)^{\frac{\alpha}{2}} u+\gamma_{1}(-\Delta)^{\frac{\alpha}{2}} v+\gamma_{2} v+r(t) \frac{F^{\prime}(u)}{\sqrt{E(u)}}=0, \\
& r_{t}=\frac{1}{2 \sqrt{E(u)}} \int_{\Omega} F^{\prime}(u) u_{t} d \mathbf{x} . \\
& u(\mathbf{x}, 0)=\phi(\mathbf{x}, y), v(\mathbf{x}, 0)=\varphi(\mathbf{x}), \mathbf{x} \in \Omega,
\end{aligned}
$$

where $E(u)=\int_{\Omega} F(u) d \mathbf{x}+C_{0}$, and $C_{0}$ is chosen such that $E(u)>0$. In addition, we assume $F \in C^{3}(\mathbb{R})$.

Theorem 3.1. (Energy dissipation) Under the periodic boundary conditions, (3.1)-(3.4) poessess the following energy function

$$
H(t)=\int_{\Omega} \frac{1}{2}\left|u_{t}\right|^{2}+\frac{\kappa}{2}\left|(-\Delta)^{\frac{\alpha}{4}} u\right|^{2} d \mathbf{x}+r^{2},
$$

and it holds that

$$
\frac{d H(t)}{d t}=-\int_{\Omega}\left(\gamma_{1}\left|(-\Delta)^{\frac{\alpha}{2}} u_{t}\right|^{2}+\gamma_{2}\left|u_{t}\right|^{2}\right) d \mathbf{x} \leq 0
$$

and thus

$$
H\left(t_{2}\right) \leq H\left(t_{1}\right), t_{1}>t_{2}
$$


Proof. Taking the inner product of (3.2) with $v$ and using (3.1), we can directly obtain

$$
\left(v_{t}, v\right)+\kappa\left((-\Delta)^{\frac{\alpha}{2}} u, u_{t}\right)+\gamma_{1}\left((-\Delta)^{\frac{\alpha}{2}} v, v\right)+\gamma_{2}(v, v)+r(t) \frac{\left(F^{\prime}(u), u_{t}\right)}{\sqrt{E(u)}}=0 .
$$

Combining with (3.1) and Leibiniz rule, we have

$$
\int_{\Omega} \frac{1}{2}\left|u_{t}\right|^{2}+\frac{\kappa}{2}\left|(-\Delta)^{\frac{\alpha}{4}} u\right|^{2} d \mathbf{x}+r(t)^{2}+\int_{\Omega}\left(\gamma_{1}\left|(-\Delta)^{\frac{\alpha}{2}} u_{t}\right|^{2}+\gamma_{2}\left|u_{t}\right|^{2}\right) d \mathbf{x}=0
$$

and one can get 3.5 and $3.6-3.7)$.

Remark 3.1. When $\gamma_{1}=\gamma_{2}=0$, the system (1.1)-(1.2) deduces to the fractional wave equation. From (3.6), we have

$$
\frac{d H(t)}{d t}=0
$$

which implies that the energy is conservative.

For a positive integer $N$, the function space is denoted by

$$
X_{N}=\operatorname{span}\left\{e^{i s x_{1}+i l x_{2}}:-N / 2 \leq s, l \leq N / 2-1\right\} .
$$

Define the orthogonal projection operator $P_{N}$ as follows

$$
\begin{aligned}
& \left(u-P_{N} u, v\right)=0, \forall v \in X_{N}, u \in L_{p e r}^{2}(\Omega), \\
& (-\Delta)^{\alpha / 2} P_{N} u=P_{N}(-\Delta)^{\alpha / 2} u .
\end{aligned}
$$

For the temporal discretization, we divide the interval $[0, T]$ by a time step size $\tau=T / K$. Let $t_{n}=n \tau, u^{n}=u\left(\mathbf{x}, t_{n}\right), 0 \leq n \leq K$, and denote

$$
\delta_{t} u^{n+\frac{1}{2}}=\frac{u^{n+1}-u^{n}}{\tau}, \tilde{u}^{n+\frac{1}{2}}=\frac{3 u^{n}-u^{n-1}}{2}, \bar{u}^{n+\frac{1}{2}}=\frac{u^{n+1}+u^{n}}{2},
$$

\subsection{The full discrete SAV Fourier spectral method}

The full discrete Fourier spectral method for (1.1)-(1.2) is given by: Find $\left(u_{N}^{n}, v_{N}^{n}\right) \in X_{N} \times X_{N}$, such that for all $(\psi, \varphi) \in X_{N} \times X_{N}$,

$$
\begin{aligned}
& \left(\delta_{t} u_{N}^{n+\frac{1}{2}}, \psi\right)=\left(\bar{v}_{N}^{n+\frac{1}{2}}, \psi\right), \\
& \left(\delta_{t} v_{N}^{n+\frac{1}{2}}, \varphi\right)+\kappa\left((-\Delta)^{\frac{\alpha}{2}} \bar{u}_{N}^{n+\frac{1}{2}}, \varphi\right)+\gamma_{1}\left((-\Delta)^{\frac{\alpha}{2}} \bar{v}_{N}^{n+\frac{1}{2}}, \varphi\right)+ \\
& \gamma_{2}\left(\bar{v}_{N}^{n+\frac{1}{2}}, \varphi\right)+\bar{R}^{n+\frac{1}{2}}\left(\frac{F^{\prime}\left(\tilde{u}_{N}^{n+\frac{1}{2}}\right)}{\sqrt{E\left(\tilde{u}_{N}^{n+\frac{1}{2}}\right)}}, \varphi\right)=0, \\
& \delta_{t} R^{n+\frac{1}{2}}=\frac{1}{2 \sqrt{E\left(\tilde{u}_{N}^{n+\frac{1}{2}}\right)}}\left(F^{\prime}\left(\tilde{u}_{N}^{n+\frac{1}{2}}\right), \delta_{t} u_{N}^{n+\frac{1}{2}}\right) \text {. }
\end{aligned}
$$




$$
u_{N}^{0}(\mathbf{x})=P_{N} u^{0}(\mathbf{x}), v_{N}^{0}(x)=P_{N} v^{0}(\mathbf{x}), R^{0}=r^{0}, \mathbf{x} \in \Omega .
$$

Since the fully discrete system (3.9)-(3.11) is not self-starting, the following scheme is used in the first step:

$$
\begin{aligned}
& \left(\frac{\tilde{u}_{N}^{\frac{1}{2}}-u_{N}^{0}}{\tau / 2}, \psi\right)=\left(\tilde{v}_{N}^{\frac{1}{2}}, \psi\right) \\
& \left(\frac{\tilde{v}_{N}^{\frac{1}{2}}-v_{N}^{0}}{\tau / 2}, \varphi\right)+\kappa\left((-\Delta)^{\frac{\alpha}{2}} \tilde{u}_{N}^{\frac{1}{2}}, \varphi\right)+\gamma_{1}\left((-\Delta)^{\frac{\alpha}{2}} \tilde{v}_{N}^{\frac{1}{2}}, \varphi\right)+\gamma_{2}\left(\tilde{v}_{N}^{\frac{1}{2}}, \varphi\right)+\tilde{R}^{\frac{1}{2}}\left(\frac{F^{\prime}\left(u_{N}^{0}\right)}{\sqrt{E\left(u_{N}^{0}\right)}}, \varphi\right)=0,(3 \\
& \frac{\tilde{R}^{\frac{1}{2}}-R^{0}}{\tau / 2}=\frac{1}{2 \sqrt{E\left(u_{N}^{0}\right)}}\left(F^{\prime}\left(u_{N}^{0}\right), \frac{\tilde{u}_{N}^{\frac{1}{2}}-u_{N}^{0}}{\tau / 2}\right)
\end{aligned}
$$

Theorem 3.2. (Unconditional full discrete energy dissipation) Under the periodic boundary conditions, (3.9)-(3.11) is dissipative in the sense that

$$
\mathbf{H}^{n} \leq \mathbf{H}^{n-1}, 1 \leq n \leq K+1,
$$

where

$$
\mathbf{H}^{n}=\frac{1}{2}\left\|v_{N}^{n}\right\|^{2}+\frac{\kappa}{2}\left\|(-\Delta)^{\frac{\alpha}{4}} u_{N}^{n}\right\|^{2}+\left(R^{n}\right)^{2}
$$

Proof. Similar to the proof for Theorem 3.1, letting $\varphi=\bar{v}_{N}^{n+\frac{1}{2}}$ in 3.10 , we have

$$
\left(\delta_{t} v_{N}^{n+\frac{1}{2}}, \bar{v}_{N}^{n+\frac{1}{2}}\right)+\kappa\left((-\Delta)^{\frac{\alpha}{2}} \bar{u}_{N}^{n+\frac{1}{2}}, \bar{v}_{N}^{n+\frac{1}{2}}\right)+\gamma_{1}\left\|(-\Delta)^{\frac{\alpha}{4}} \bar{v}_{N}^{n+\frac{1}{2}}\right\|^{2}+\gamma_{2}\left\|\bar{v}_{N}^{n+\frac{1}{2}}\right\|^{2}+\bar{R}^{n+\frac{1}{2}} \frac{\left(F^{\prime}\left(\tilde{u}_{N}^{n+\frac{1}{2}}\right), \bar{v}_{N}^{n+\frac{1}{2}}\right)}{\sqrt{E\left(\tilde{u}_{N}^{n+\frac{1}{2}}\right)}}=0 .
$$

Taking $\psi=(-\Delta)^{\frac{\alpha}{2}} \bar{u}_{N}^{n+\frac{1}{2}}$ in $(3.9)$, it yields

$$
\left(\delta_{t} u_{N}^{n+\frac{1}{2}},(-\Delta)^{\frac{\alpha}{2}} \bar{u}_{N}^{n+\frac{1}{2}}\right)=\left(\bar{v}_{N}^{n+\frac{1}{2}},(-\Delta)^{\frac{\alpha}{2}} \bar{u}_{N}^{n+\frac{1}{2}}\right) .
$$

Using

$$
\left(\delta_{t} v_{N}^{n+\frac{1}{2}}, \bar{v}_{N}^{n+\frac{1}{2}}\right)=\frac{1}{2 \tau}\left(\left\|v_{N}^{n+1}\right\|^{2}-\left\|v_{N}^{n}\right\|^{2}\right),\left(\delta_{t} u_{N}^{n+\frac{1}{2}},(-\Delta)^{\frac{\alpha}{2}} \bar{u}_{N}^{n+\frac{1}{2}}\right)=\frac{1}{2 \tau}\left(\left|u_{N}^{n+1}\right|^{2}-\left|u_{N}^{n}\right|^{2}\right)
$$

and (3.11), it directly achieves $\left(\frac{1}{2}\left\|v_{N}^{n+1}\right\|^{2}+\frac{\kappa}{2}\left\|(-\Delta)^{\frac{\alpha}{4}} u_{N}^{n+1}\right\|^{2}+\left(R^{n+1}\right)^{2}\right)-\left(\frac{1}{2}\left\|v_{N}^{n}\right\|^{2}+\frac{\kappa}{2}\left\|(-\Delta)^{\frac{\alpha}{4}} u_{N}^{n}\right\|^{2}+\left(R^{n}\right)^{2}\right)+\tau \gamma_{1}\left\|(-\Delta)^{\frac{\alpha}{4}} \bar{v}_{N}^{n+\frac{1}{2}}\right\|^{2}+\tau \gamma_{2}\left\|\bar{v}_{N}^{n+\frac{1}{2}}\right\|^{2}=0$. 
Throughout the paper, denote $\mathrm{C}$ a generic positive constant independent of $\mathrm{K}$ and $\mathrm{N}$ that may has different values in different cases. We assume that

$\left\|u_{0}\right\|_{H^{m}}+\|u\|_{H^{m}}+|r|_{L^{\infty}(0, T)}+\left|r_{t}\right|_{L^{\infty}(0, T)}+\left|r_{t t}\right|_{L^{\infty}(0, T)}+\left|r_{t t t}\right|_{L^{\infty}(0, T)}+\left\|u_{t}\right\|_{H^{m}}+\|u\|_{\alpha}+\left\|u_{t}\right\|_{\alpha}+\left\|u_{t t}\right\|_{\alpha}+\left\|u_{t t t}\right\|_{\alpha / 2} \leq L$,

where $L$ is a positive constant independent of $N$ and $\tau$.

With the assumption, we present unconditional optimal error estimates for the FGWE in the following theorem. For simplicity, we assume $u^{n}:=u\left(x, t_{n}\right)$.

Theorem 3.3. Let $u$ and $\left\{u_{N}^{n}\right\}_{n=0}^{K}$ be the solutions of (1.1)-(1.2) and (3.9)-(3.15), respectively. Assume u satisfies (3.19). Then it holds that

$$
\left\|u^{n}-u_{N}^{n}\right\|^{2}+\left\|v^{n}-v_{N}^{n}\right\|^{2}+\left|r^{n}-R^{n}\right|^{2} \leq C\left(\tau^{4}+N^{-2 m}\right), \kappa\left|u^{n}-u_{N}^{n}\right|_{\frac{\alpha}{2}}^{2} \leq C\left(\tau^{4}+N^{\alpha-2 m}\right),
$$

where $C$ are positive constants independent of $N$ and $\tau$.

Taking technique similar as [40], we prove the Theorem 3.3 in the next two sections. We splitting error into two parts, i.e., temporal error and spatial error.

\section{Error analysis for the time-discrete system}

In this section, we present and analyze the time-discrete system for the nonlinear FGWE. We get the following time-discrete scheme based on SAV approach, which define $U^{n}, V^{n}$ and $R^{n}$ such that

$$
\begin{aligned}
& \delta_{t} U^{n+\frac{1}{2}}=\bar{V}^{n+\frac{1}{2}} \\
& \delta_{t} V^{n+\frac{1}{2}}+\kappa(-\Delta)^{\frac{\alpha}{2}} \bar{U}^{n+\frac{1}{2}}+\gamma_{1}(-\Delta)^{\frac{\alpha}{2}} \bar{V}^{n+\frac{1}{2}}+\gamma_{2} \bar{V}^{n+\frac{1}{2}}+\bar{R}^{n+\frac{1}{2}} \frac{F^{\prime}\left(\tilde{U}^{n+\frac{1}{2}}\right)}{\sqrt{E\left(\tilde{U}^{n+\frac{1}{2}}\right)}}=0, \\
& \delta_{t} R^{n+\frac{1}{2}}=\frac{1}{2 \sqrt{E\left(\tilde{U}^{n+\frac{1}{2}}\right)}}\left(F^{\prime}\left(\tilde{U}^{n+\frac{1}{2}}\right), \delta_{t} U^{n+\frac{1}{2}}\right), \\
& U^{0}(\mathbf{x})=u^{0}(\mathbf{x}), V^{0}(x)=v^{0}(\mathbf{x}), R^{0}=r^{0}, \mathbf{x} \in \Omega .
\end{aligned}
$$

While the first step is obtained by

$$
\begin{aligned}
& \frac{\tilde{U}^{\frac{1}{2}}-U^{0}}{\tau / 2}=\tilde{V}^{\frac{1}{2}} \\
& \frac{\tilde{V}^{\frac{1}{2}}-V^{0}}{\tau / 2}+\kappa(-\Delta)^{\frac{\alpha}{2}} \tilde{U}^{\frac{1}{2}}+\gamma_{1}(-\Delta)^{\frac{\alpha}{2}} \tilde{V}^{\frac{1}{2}}+\gamma_{2} \tilde{V}^{\frac{1}{2}}+\tilde{R}^{\frac{1}{2}} \frac{F^{\prime}\left(U^{0}\right)}{\sqrt{E\left(U^{0}\right)}}=0 \\
& \frac{\tilde{R}^{\frac{1}{2}}-R^{0}}{\tau / 2}=\frac{1}{2 \sqrt{E\left(U^{0}\right)}}\left(F^{\prime}\left(U^{0}\right), \frac{\tilde{U}^{\frac{1}{2}}-U^{0}}{\tau / 2}\right) .
\end{aligned}
$$


By using the 3.8$)$ and denote $\tilde{b}^{n}:=\frac{F^{\prime}\left(\tilde{U}^{n+\frac{1}{2}}\right)}{\sqrt{E\left(\tilde{U}^{n+\frac{1}{2}}\right)}}$, we can get a linear equation from the SAV scheme (4.1)- 4.3,

$$
A U^{n+1}+\frac{\tau^{2}}{4}\left(\tilde{b}^{n}, U^{n+1}\right) \tilde{b}^{n}=g^{n},
$$

where $A=\left(2+\tau \gamma_{2}\right) I+\left(\frac{\tau^{2}}{2} \kappa+\tau \gamma_{1}\right)(-\Delta)^{\alpha / 2}, g^{n}=\left(A-\tau^{2} \kappa(-\Delta)^{\alpha / 2}\right) U^{n}+2 \tau V^{n}+\frac{\tau^{2}}{4}\left(\tilde{b}^{n}, U^{n}\right) \tilde{b}^{n}-\tau^{2} R^{n} \tilde{b}^{n}$. We can solve above linear equation by first computing $A^{-1} \tilde{b}^{n}$ and $A^{-1} g^{n}$. Thus, it leads to

$$
U^{n+1}+\frac{\tau^{2}}{4}\left(\tilde{b}^{n}, U^{n+1}\right) A^{-1} \tilde{b}^{n}=A^{-1} g^{n} .
$$

Taking the inner product with $\tilde{b}^{n}$ on the both sides of the above resulting equation to solve $\left(\tilde{b}^{n}, U^{n+1}\right)$, then, we can get $U^{n+1}$. Similar to the same solving procedure, we can get $\tilde{U}^{\frac{1}{2}}$ from the Eqs. (4.5)(4.7) (see [31] for more details). In summary, we solve the schemes (4.1)-(4.3) by the following main procedure at each time step and the solving procedure of schemes 4.5 - 4.7 is similar.

1. Assume $U^{n}, R^{n}, U^{n-1}, V^{n}$ and $R^{n-1}$ are known;

2. Compute $A^{-1} \tilde{b}^{n}$ and $A^{-1} g^{n}$;

3. Compute $\left(\tilde{b}^{n}, U^{n+1}\right)$ by solving the resulting equation, which is derived from taking the inner product with $\tilde{b}^{n}$ on the both sides of 4.9$]$;

4. Solve 4.8) to obtain $U^{n+1}$;

5. Compute $V^{n+1}$ by solving (4.1);

6. Solve 4.3 to get $R^{n+1}$.

Theorem 4.1. (Unconditional energy dissipation) Under the periodic boundary conditions, 4.1)(4.3) is dissipative in the sense that

$$
\mathbb{H}^{n+1} \leq \mathbb{H}^{n}, 0 \leq n \leq K
$$

where

$$
\mathbb{H}^{n}=\int_{\Omega} \frac{1}{2}\left|V^{n}\right|^{2}+\frac{\kappa}{2}\left|(-\Delta)^{\frac{\alpha}{4}} U^{n}\right|^{2} d \mathbf{x}+\left(R^{n}\right)^{2} .
$$

Proof. Taking the inner product of (4.2) with $\bar{V}^{n+\frac{1}{2}}$, combining with (4.1), we get $\left(\delta_{t} V^{n+\frac{1}{2}}, \bar{V}^{n+\frac{1}{2}}\right)+\kappa\left((-\Delta)^{\frac{\alpha}{2}} \bar{U}^{n+\frac{1}{2}}, \delta_{t} U^{n+\frac{1}{2}}\right)+\gamma_{1}\left\|(-\Delta)^{\frac{\alpha}{4}} \bar{V}^{n+\frac{1}{2}}\right\|^{2}+\gamma_{2}\left\|\bar{V}^{n+\frac{1}{2}}\right\|^{2}+\bar{R}^{n+\frac{1}{2}} \frac{\left(F^{\prime}\left(\tilde{U}^{n+\frac{1}{2}}\right), \bar{V}^{n+\frac{1}{2}}\right)}{\sqrt{E\left(\tilde{U}^{n+\frac{1}{2}}\right)}}=0$.

By using (4.3) and

$$
\left(\delta_{t} V^{n+\frac{1}{2}}, \bar{V}^{n+\frac{1}{2}}\right)=\frac{1}{2 \tau}\left(\left\|V^{n+1}\right\|^{2}-\left\|V^{n}\right\|^{2}\right),\left((-\Delta)^{\frac{\alpha}{2}} \bar{U}^{n+\frac{1}{2}}, \delta_{t} U^{n+\frac{1}{2}}\right)=\frac{1}{2 \tau}\left(\left\|(-\Delta)^{\frac{\alpha}{4}} U^{n+1}\right\|^{2}-\left\|(-\Delta)^{\frac{\alpha}{4}} U^{n}\right\|^{2}\right),
$$

we easily obtain 4.10 . 


\subsection{Error estimate for time discrete scheme}

Denote $e_{u}^{n}=u^{n}-U^{n}, e_{v}^{n}=v^{n}-V^{n}$ and $e_{r}^{n}=r^{n}-R^{n}$.

Theorem 4.2. Suppose that (1.1)-(1.2) has the unique solution $(u, v, r) \in H_{p e r}^{\alpha}(\Omega) \times H_{p e r}^{\alpha}(\Omega) \times$ $C^{3}(0, T)$. The assumption (3.19) and $U^{0} \in H^{\alpha}$ hold. Then for $0 \leq n \leq K$, there exists a positive constant $\tau_{1}^{*}$, such that (4.1)-(4.7) admits unique solution $\left(U^{n}, V^{n}\right) \in H_{p e r}^{\alpha}(\Omega) \times H_{p e r}^{\alpha}(\Omega)$ and for $\tau<\tau_{1}^{*}$

$$
\begin{aligned}
& \left\|e_{u}^{n}\right\|^{2}+\kappa\left|e_{u}^{n}\right|_{\frac{\alpha}{2}}^{2}+\kappa\left|e_{u}^{n}\right|_{\alpha}^{2}+\left\|e_{v}^{n}\right\|^{2}+\left|e_{v}^{n}\right|_{\frac{\alpha}{2}}^{2}+\left(e_{r}^{n}\right)^{2} \leq C \tau^{3}, n=0, \ldots, K, \\
& \left\|U^{n}\right\|_{\infty} \leq M, n=0, \ldots, K,
\end{aligned}
$$

where $M>0$ is a bounded constant independent of $K$.

Proof. The (4.1)-(4.7) can be rewritten as the following elliptic systems

$$
\left\{\begin{array}{l}
\frac{2 \delta_{t} U^{n+\frac{1}{2}}-2 V^{n}}{\tau}+\kappa(-\Delta)^{\frac{\alpha}{2}} \bar{U}^{n+\frac{1}{2}}+\gamma_{1}(-\Delta)^{\frac{\alpha}{2}} \delta_{t} U^{n+\frac{1}{2}}+\gamma_{2} \delta_{t} U^{n+\frac{1}{2}}+\bar{R}^{n+\frac{1}{2}} \frac{F^{\prime}\left(\tilde{U}^{n+\frac{1}{2}}\right)}{\sqrt{E\left(\tilde{U}^{n+\frac{1}{2}}\right)}}=0, \\
\delta_{t} R^{n+\frac{1}{2}}=\frac{1}{2 \sqrt{E\left(\tilde{U}^{n+\frac{1}{2}}\right)}}\left(F^{\prime}\left(\tilde{U}^{n+\frac{1}{2}}\right), \delta_{t} U^{n+\frac{1}{2}}\right), \forall n=1,2, \cdots, K, \\
\frac{\tilde{U}^{\frac{1}{2}}-U^{0}-\frac{\tau}{2} V^{0}}{\frac{\tau^{2}}{4}}+\kappa(-\Delta)^{\frac{\alpha}{2}} \tilde{U}^{\frac{1}{2}}+\gamma_{1}(-\Delta)^{\frac{\alpha}{2}} \frac{\tilde{U}^{\frac{1}{2}}-U^{0}}{\tau / 2}+\gamma_{2} \frac{\tilde{U}^{\frac{1}{2}}-U^{0}}{\tau / 2}+\tilde{R}^{\frac{1}{2}} \frac{F^{\prime}\left(U^{0}\right)}{\sqrt{E\left(U^{0}\right)}}=0, \\
\frac{\tilde{R}^{\frac{1}{2}}-R^{0}}{\tau / 2}=\frac{1}{2 \sqrt{E\left(U^{0}\right)}}\left(F^{\prime}\left(U^{0}\right), \frac{\tilde{U}^{\frac{1}{2}}-U^{0}}{\tau / 2}\right) .
\end{array}\right.
$$

The existence and uniqueness of the solution to the linear elliptic equations are straightforward. The (3.1)-(3.3) for time discretization at $t_{n+\frac{1}{2}}$ follow that

$$
\begin{aligned}
& \delta_{t} u^{n+\frac{1}{2}}=\bar{v}^{n+\frac{1}{2}}+Q_{1}^{n+\frac{1}{2}}, \\
& \delta_{t} v^{n+\frac{1}{2}}+\kappa(-\Delta)^{\frac{\alpha}{2}} \bar{u}^{n+\frac{1}{2}}+\gamma_{1}(-\Delta)^{\frac{\alpha}{2}} \bar{v}^{n+\frac{1}{2}}+\gamma_{2} \bar{v}^{n+\frac{1}{2}}+\bar{r}^{n+\frac{1}{2}} \frac{F^{\prime}\left(\tilde{u}^{n+\frac{1}{2}}\right)}{\sqrt{E\left(\tilde{u}^{n+\frac{1}{2}}\right)}}=Q_{2}^{n+\frac{1}{2}}, \\
& \delta_{t} r^{n+\frac{1}{2}}=\frac{1}{2 \sqrt{E\left(\tilde{u}^{n+\frac{1}{2}}\right)}}\left(F^{\prime}\left(\tilde{u}^{n+\frac{1}{2}}\right), \delta_{t} u^{n+\frac{1}{2}}\right)+Q_{3}^{n+\frac{1}{2}},
\end{aligned}
$$

where

$$
\begin{aligned}
Q_{1}^{n+\frac{1}{2}} & =\left(\delta_{t} u^{n+\frac{1}{2}}-u_{t}^{n+\frac{1}{2}}\right)-\left(\bar{v}^{n+\frac{1}{2}}-v^{n+\frac{1}{2}}\right), \\
Q_{2}^{n+\frac{1}{2}} & \left.=\left(\delta_{t} v^{n+\frac{1}{2}}-v_{t}^{n+\frac{1}{2}}\right)+\kappa\left((-\Delta)^{\frac{\alpha}{2}} \bar{u}^{n+\frac{1}{2}}-(-\Delta)^{\frac{\alpha}{2}} u^{n+\frac{1}{2}}\right)+\gamma_{1}\left((-\Delta)^{\frac{\alpha}{2}} \bar{v}^{n+\frac{1}{2}}-(-\Delta)^{\frac{\alpha}{2}} v^{n+\frac{1}{2}}\right]\right) \\
& +\gamma_{2}\left(\bar{v}^{n+\frac{1}{2}}-v^{n+\frac{1}{2}}\right)+\left(\bar{r}^{n+\frac{1}{2}} \frac{F^{\prime}\left(\tilde{u}^{n+\frac{1}{2}}\right)}{\sqrt{E\left(\tilde{u}^{n+\frac{1}{2}}\right)}}-r^{n+\frac{1}{2}} \frac{F^{\prime}\left(u^{n+\frac{1}{2}}\right)}{\sqrt{E\left(u^{n+\frac{1}{2}}\right)}}\right),
\end{aligned}
$$




$$
Q_{3}^{n+\frac{1}{2}}=\left(\delta_{t} r^{n+\frac{1}{2}}-r_{t}^{n+\frac{1}{2}}\right)-\left(\frac{1}{2 \sqrt{E\left(\tilde{u}^{n+\frac{1}{2}}\right)}}\left(F^{\prime}\left(\tilde{u}^{n+\frac{1}{2}}\right), \delta_{t} u^{n+\frac{1}{2}}\right)-\frac{1}{2 \sqrt{E\left(u^{n+\frac{1}{2}}\right)}} \int_{\Omega} F^{\prime}\left(u^{n+\frac{1}{2}}\right) u_{t}^{n+\frac{1}{2}} d \mathbf{x}\right) .
$$

By (3.1)-(3.3) for time discretization at $t_{\frac{1}{2}}$, it follows that

$$
\begin{aligned}
& \frac{\tilde{u}^{\frac{1}{2}}-u^{0}}{\tau / 2}=\tilde{v}^{\frac{1}{2}}+\tilde{Q}_{1}^{\frac{1}{2}}, \\
& \frac{\tilde{v}^{\frac{1}{2}}-v^{0}}{\tau / 2}+\kappa(-\Delta)^{\frac{\alpha}{2}} \tilde{u}^{\frac{1}{2}}+\gamma_{1}(-\Delta)^{\frac{\alpha}{2}} \tilde{v}^{\frac{1}{2}}+\gamma_{2} \tilde{v}^{\frac{1}{2}}+\tilde{r}^{\frac{1}{2}} \frac{F^{\prime}\left(u^{0}\right)}{\sqrt{E\left(u^{0}\right)}}=\tilde{Q}_{2}^{\frac{1}{2}}, \\
& \frac{\tilde{r}^{\frac{1}{2}}-r^{0}}{\tau / 2}=\frac{1}{2 \sqrt{E\left(u^{0}\right)}}\left(F^{\prime}\left(u^{0}\right), \frac{\tilde{u}^{\frac{1}{2}}-u^{0}}{\tau / 2}\right)+\tilde{Q}_{3}^{\frac{1}{2}},
\end{aligned}
$$

where

$$
\begin{aligned}
\tilde{Q}_{1}^{\frac{1}{2}} & =\left(\frac{\tilde{u}^{\frac{1}{2}}-u^{0}}{\tau / 2}-u_{t}^{\frac{1}{2}}\right)-\left(\tilde{v}^{\frac{1}{2}}-v^{\frac{1}{2}}\right), \\
\tilde{Q}_{2}^{\frac{1}{2}} & =\left(\frac{\tilde{v}^{\frac{1}{2}}-v^{0}}{\tau / 2}-v_{t}^{\frac{1}{2}}\right)+\kappa\left((-\Delta)^{\frac{\alpha}{2}} \tilde{u}^{\frac{1}{2}}-(-\Delta)^{\frac{\alpha}{2}} u^{\frac{1}{2}}\right)+\gamma_{1}\left((-\Delta)^{\frac{\alpha}{2}} \tilde{v}^{\frac{1}{2}}-(-\Delta)^{\frac{\alpha}{2}} v^{\frac{1}{2}}\right) \\
& +\gamma_{2}\left(\tilde{v}^{\frac{1}{2}}-v^{\frac{1}{2}}\right)+\left(\tilde{r}^{\frac{1}{2}} \frac{F^{\prime}\left(\tilde{u}^{0}\right)}{\sqrt{E\left(\tilde{u}^{0)}\right.}}-r^{\frac{1}{2}} \frac{F^{\prime}\left(u^{\frac{1}{2}}\right)}{\sqrt{E\left(u^{\frac{1}{2}}\right)}}\right), \\
\tilde{Q}_{3}^{\frac{1}{2}}= & \left(\frac{\tilde{r}^{\frac{1}{2}}-r^{0}}{\tau / 2}-r_{t}^{\frac{1}{2}}\right)-\left(\frac{1}{2 \sqrt{E\left(u^{0}\right)}}\left(F^{\prime}\left(u^{0}\right), \frac{\tilde{u}^{\frac{1}{2}}-u^{0}}{\tau / 2}\right)-\frac{1}{2 \sqrt{E\left(u^{\frac{1}{2}}\right)}} \int_{\Omega} F^{\prime}\left(u^{n+\frac{1}{2}}\right) u_{t}^{n+\frac{1}{2}}\right) .
\end{aligned}
$$

Using Taylor formula, we get

$$
\tau \sum_{n=0}^{K}\left(\left\|Q_{1}^{n+\frac{1}{2}}\right\|+\left\|Q_{2}^{n+\frac{1}{2}}\right\|+\left\|Q_{3}^{n+\frac{1}{2}}\right\|\right)+\tau\left(\left\|\tilde{Q}_{1}^{\frac{1}{2}}\right\|+\left\|\tilde{Q}_{2}^{\frac{1}{2}}\right\|+\left\|\tilde{Q}_{3}^{\frac{1}{2}}\right\|\right) \leq C \tau^{2} .
$$

We will give the proof sketch for (4.12)-(4.13).

1. The first step: error estimate for $\tilde{e}_{u}^{\frac{1}{2}}=\tilde{u}^{\frac{1}{2}}-\tilde{U}^{\frac{1}{2}}, \tilde{e}_{v}^{\frac{1}{2}}=\tilde{v}^{\frac{1}{2}}-\tilde{V}^{\frac{1}{2}}, \tilde{e}_{r}^{\frac{1}{2}}=\tilde{r}^{\frac{1}{2}}-\tilde{R}^{\frac{1}{2}}$.

Combining (4.17)-(4.19) and (4.5)-(4.7), we arrive at

$$
\begin{aligned}
& \frac{\tilde{e}_{u}^{\frac{1}{2}}}{\tau / 2}=\tilde{e}_{v}^{\frac{1}{2}}+\tilde{Q}_{1}^{\frac{1}{2}}, \\
& \frac{\tilde{e}_{v}^{\frac{1}{2}}}{\tau / 2}+\kappa(-\Delta)^{\frac{\alpha}{2}} \tilde{e}_{u}^{\frac{1}{2}}+\gamma_{1}(-\Delta)^{\frac{\alpha}{2}} \tilde{e}_{v}^{\frac{1}{2}}+\gamma_{2} \tilde{e}_{v}^{\frac{1}{2}}+\tilde{G}^{\frac{1}{2}}=\tilde{Q}_{2}^{\frac{1}{2}}, \\
& \frac{\tilde{e}_{r}^{\frac{1}{2}}}{\tau / 2}=\tilde{F}^{\frac{1}{2}}+\tilde{Q}_{3}^{\frac{1}{2}},
\end{aligned}
$$


where

$$
\tilde{G}^{\frac{1}{2}}=\tilde{r}^{\frac{1}{2}} \frac{F^{\prime}\left(u^{0}\right)}{\sqrt{E\left(u^{0}\right)}}-\tilde{R}^{\frac{1}{2}} \frac{F^{\prime}\left(U^{0}\right)}{\sqrt{E\left(U^{0}\right)}}
$$

and

$$
\tilde{F}^{\frac{1}{2}}=\frac{1}{2 \sqrt{E\left(u^{0}\right)}}\left(F^{\prime}\left(u^{0}\right), \frac{\tilde{u}^{\frac{1}{2}}-u^{0}}{\tau / 2}\right)-\frac{1}{2 \sqrt{E\left(U^{0}\right)}}\left(F^{\prime}\left(U^{0}\right), \frac{\tilde{U}^{\frac{1}{2}}-U^{0}}{\tau / 2}\right) .
$$

Taking the inner product of $\tilde{e}_{u}^{\frac{1}{2}}, \tilde{e}_{v}^{\frac{1}{2}}$ in $(4.21)$ and $(4.22)$ respectively, as well as multiplying $\tilde{e}_{r}^{\frac{1}{2}}$ on the both sides of (4.23).

$$
\begin{aligned}
& \left\|\tilde{e}_{u}^{\frac{1}{2}}\right\|^{2}=\frac{\tau}{2}\left(\tilde{e}_{u}^{\frac{1}{2}}, \tilde{e}_{v}^{\frac{1}{2}}\right)+\frac{\tau}{2}\left(\tilde{Q}_{1}^{\frac{1}{2}}, \tilde{e}_{u}^{\frac{1}{2}}\right) \\
& \left\|\tilde{e}_{v}^{\frac{1}{2}}\right\|^{2}+\frac{\tau}{2} \kappa\left((-\Delta)^{\frac{\alpha}{2}} \tilde{e}_{u}^{\frac{1}{2}}, \tilde{e}_{v}^{\frac{1}{2}}\right)+\frac{\tau}{2} \gamma_{1}\left|\tilde{e}_{v}^{\frac{1}{2}}\right|_{\frac{\alpha}{2}}^{2}+\frac{\tau}{2} \gamma_{2}\left\|\tilde{e}_{v}^{\frac{1}{2}}\right\|^{2}=-\frac{\tau}{2}\left(\tilde{G}^{\frac{1}{2}}, \tilde{e}_{v}^{\frac{1}{2}}\right)+\frac{\tau}{2}\left(\tilde{Q}_{2}^{\frac{1}{2}}, \tilde{e}_{v}^{\frac{1}{2}}\right), \\
& \left(\tilde{e}_{r}^{\frac{1}{2}}\right)^{2}=\frac{\tau}{2}\left(\tilde{F}^{\frac{1}{2}}, \tilde{e}_{r}^{\frac{1}{2}}\right)+\frac{\tau}{2}\left(\tilde{Q}_{3}^{\frac{1}{2}}, \tilde{e}_{r}^{\frac{1}{2}}\right) .
\end{aligned}
$$

Using (4.21), the 4.25) can be rewritten as

$$
\left\|\tilde{e}_{v}^{\frac{1}{2}}\right\|^{2}+\kappa\left|\tilde{e}_{u}^{\frac{1}{2}}\right|_{\frac{\alpha}{2}}^{2}+\frac{\tau}{2} \gamma_{1}\left|\tilde{e}_{v}^{\frac{1}{2}}\right|_{\frac{\alpha}{2}}^{2}+\frac{\tau}{2} \gamma_{2}\left\|\tilde{e}_{v}^{\frac{1}{2}}\right\|^{2}=-\frac{\tau}{2}\left(\tilde{G}^{\frac{1}{2}}, \tilde{e}_{v}^{\frac{1}{2}}\right)+\frac{\tau}{2}\left(\tilde{Q}_{2}^{\frac{1}{2}}, \tilde{e}_{v}^{\frac{1}{2}}\right)+\frac{\tau}{2} \kappa\left((-\Delta)^{\frac{\alpha}{2}} \tilde{e}_{u}^{\frac{1}{2}}, \tilde{Q}_{1}^{\frac{1}{2}}\right) .
$$

In addition, $\tilde{G}^{\frac{1}{2}}$ and $\tilde{F}^{\frac{1}{2}}$ could be represented as

$$
\begin{aligned}
& \tilde{G}^{\frac{1}{2}}=\tilde{r}^{\frac{1}{2}}\left(\frac{F^{\prime}\left(u^{0}\right)}{\sqrt{E\left(u^{0}\right)}}-\frac{F^{\prime}\left(U^{0}\right)}{\sqrt{E\left(U^{0}\right)}}\right)+\tilde{e}_{r}^{\frac{1}{2}} \frac{F^{\prime}\left(U^{0}\right)}{\sqrt{E\left(U^{0}\right)}}, \\
& \tilde{F}^{\frac{1}{2}}=\left(\frac{1}{2 \sqrt{E\left(u^{0}\right)}} F^{\prime}\left(u^{0}\right)-\frac{1}{2 \sqrt{E\left(U^{0}\right)}} F^{\prime}\left(U^{0}\right), \frac{\tilde{U}^{\frac{1}{2}}-U^{0}}{\tau / 2}\right)+\left(\frac{1}{2 \sqrt{E\left(u^{0}\right)}} F^{\prime}\left(u^{0}\right), \frac{\tilde{e}_{u}^{\frac{1}{2}}}{\tau / 2}\right) .
\end{aligned}
$$

From (4.24)-4.26 and Lemma 2.1, using (4.27)-4.29) and using the Cauchy-Schwarz inequality and Young's inequality, we obtain

$$
\begin{aligned}
& \left\|\tilde{e}_{u}^{\frac{1}{2}}\right\|^{2} \leq C \tau\left\|\tilde{e}_{u}^{\frac{1}{2}}\right\|^{2}+C \tau\left\|\tilde{Q}_{1}^{\frac{1}{2}}\right\|^{2}+C \tau\left\|\tilde{e}_{v}^{\frac{1}{2}}\right\|^{2}, \\
& \begin{aligned}
\left\|\tilde{e}_{v}^{\frac{1}{2}}\right\|^{2}+\kappa\left|\tilde{e}_{u}^{\frac{1}{2}}\right|_{\frac{\alpha}{2}}^{2}+\frac{\tau}{2} \gamma_{1}\left|\tilde{e}_{v}^{\frac{1}{2}}\right|_{\frac{\alpha}{2}}^{2}+\frac{\tau}{2} \gamma_{2}\left\|\tilde{e}_{v}^{\frac{1}{2}}\right\|^{2} \leq C \tau\left(\tilde{e}_{r}^{\frac{1}{2}}\right)^{2}+C \tau\left\|\tilde{e}_{v}^{\frac{1}{2}}\right\|^{2}+C \tau\left\|\tilde{Q}_{2}^{\frac{1}{2}}\right\|^{2} \\
+C \tau\left|\tilde{e}_{u}^{\frac{1}{2}}\right|_{\frac{\alpha}{2}}^{2}+C \tau\left|\tilde{Q}_{1}^{\frac{1}{2}}\right|_{\frac{\alpha}{2}}^{2},
\end{aligned} \\
& \left(\tilde{e}_{r}^{\frac{1}{2}}\right)^{2} \leq C \tau\left\|\tilde{e}_{v}^{\frac{1}{2}}\right\|^{2}+C \tau\left(\tilde{e}_{r}^{\frac{1}{2}}\right)^{2}+C \tau\left\|\tilde{Q}_{3}^{\frac{1}{2}}\right\|^{2}+C \tau\left\|\tilde{Q}_{1}^{\frac{1}{2}}\right\|^{2} .
\end{aligned}
$$

By combining the above equations, we can obtain

$$
\left\|\tilde{e}_{u}^{\frac{1}{2}}\right\|^{2}+\kappa\left|\tilde{e}_{u}^{\frac{1}{2}}\right|_{\frac{\alpha}{2}}^{2}+\left\|\tilde{e}_{v}^{\frac{1}{2}}\right\|^{2}+\left(\tilde{e}_{r}^{\frac{1}{2}}\right)^{2} \leq C \tau\left(\left|\tilde{Q}_{1}^{\frac{1}{2}}\right|_{\frac{\alpha}{2}}^{2}+\left\|\tilde{Q}_{2}^{\frac{1}{2}}\right\|^{2}+\left\|\tilde{Q}_{3}^{\frac{1}{2}}\right\|^{2}\right) \leq C \tau^{3} .
$$

Moreover, take the inner product of 4.22 by $(-\Delta)^{\frac{\alpha}{2}} \tilde{e}_{v}^{\frac{1}{2}}$ to get

$$
\left|\tilde{e}_{v}^{\frac{1}{2}}\right|_{\frac{\alpha}{2}}^{2}+\frac{\tau}{2} \kappa\left((-\Delta)^{\frac{\alpha}{2}} \tilde{e}_{u}^{\frac{1}{2}},(-\Delta)^{\frac{\alpha}{2}} \tilde{e}_{v}^{\frac{1}{2}}\right)+\frac{\tau}{2} \gamma_{1}\left|\tilde{e}_{v}^{\frac{1}{2}}\right|_{\alpha}^{2}+\frac{\tau}{2} \gamma_{2}\left|\tilde{e}_{v}^{\frac{1}{2}}\right|_{\frac{\alpha}{2}}^{2}=-\frac{\tau}{2}\left(\tilde{G}^{\frac{1}{2}}+\tilde{Q}_{2}^{\frac{1}{2}},(-\Delta)^{\frac{\alpha}{2}} \tilde{e}_{v}^{\frac{1}{2}}\right) .
$$


Using $\frac{(-\Delta)^{\frac{\alpha}{2}} \tilde{e}_{u}^{\frac{1}{2}}}{\tau / 2}=(-\Delta)^{\frac{\alpha}{2}} \tilde{e}_{v}^{\frac{1}{2}}+(-\Delta)^{\frac{\alpha}{2}} \tilde{Q}_{1}^{\frac{1}{2}}$, 4.34) can be rewritten as $\left|\tilde{e}_{v}^{\frac{1}{2}}\right|_{\frac{\alpha}{2}}^{2}+\kappa\left|\tilde{e}_{u}^{\frac{1}{2}}\right|_{\alpha}^{2}+\frac{\tau}{2} \gamma_{1}\left|\tilde{e}_{v}^{\frac{1}{2}}\right|_{\alpha}^{2}+\frac{\tau}{2} \gamma_{2}\left|\tilde{e}_{v}^{\frac{1}{2}}\right|_{\frac{\alpha}{2}}^{2} \leq \frac{\tau}{2}\left|\left(\tilde{G}^{\frac{1}{2}},(-\Delta)^{\frac{\alpha}{2}} \tilde{e}_{v}^{\frac{1}{2}}\right)+\left(\tilde{Q}_{2}^{\frac{1}{2}},(-\Delta)^{\frac{\alpha}{2}} \tilde{e}_{v}^{\frac{1}{2}}\right)+\kappa\left((-\Delta)^{\frac{\alpha}{2}} \tilde{Q}_{1}^{\frac{1}{2}},(-\Delta)^{\frac{\alpha}{2}} \tilde{e}_{u}^{\frac{1}{2}}\right)\right|$.

From Lemma 2.1, the three terms on the right side of the above equation can be controlled by

$$
\begin{aligned}
& \left|\left(\tilde{G}^{\frac{1}{2}},(-\Delta)^{\frac{\alpha}{2}} \tilde{e}_{v}^{\frac{1}{2}}\right)\right| \leq\left|\tilde{e}_{r}^{\frac{1}{2}}\left(\frac{1}{\sqrt{E\left(U^{0}\right)}} F^{\prime}\left(U^{0}\right),(-\Delta)^{\frac{\alpha}{2}} \tilde{e}_{v}^{\frac{1}{2}}\right)\right| \leq C(L)\left(\tilde{e}_{r}^{\frac{1}{2}}\right)^{2}+\frac{c_{1}}{2}\left|\tilde{e}_{v}^{\frac{1}{2}}\right|_{\frac{\alpha}{2}}^{2}, \\
& \left|\left(\tilde{Q}_{2}^{\frac{1}{2}},(-\Delta)^{\frac{\alpha}{2}} \tilde{e}_{v}^{\frac{1}{2}}\right)\right| \leq \frac{c_{2}}{2}\left|\tilde{e}_{v}^{\frac{1}{2}}\right|_{\frac{\alpha}{2}}^{2}+\frac{c_{3}}{2}\left|\tilde{Q}_{2}^{\frac{1}{2}}\right|_{\frac{\alpha}{2}}^{2}, \\
& \left|\left((-\Delta)^{\frac{\alpha}{2}} \tilde{Q}_{1}^{\frac{1}{2}},(-\Delta)^{\frac{\alpha}{2}} \tilde{e}_{u}^{\frac{1}{2}}\right)\right| \leq \frac{c_{4}}{2}\left|\tilde{e}_{u}^{\frac{1}{2}}\right|_{\alpha}^{2}+\frac{c_{5}}{2}\left|\tilde{Q}_{1}^{\frac{1}{2}}\right|_{\alpha}^{2} .
\end{aligned}
$$

From (4.33) and 4.35)- (4.37), then we can get

$$
\left\|\tilde{e}_{u}^{\frac{1}{2}}\right\|^{2}+\kappa\left|\tilde{e}_{u}^{\frac{1}{2}}\right|_{\frac{\alpha}{2}}^{2}+\left\|\tilde{e}_{v}^{\frac{1}{2}}\right\|^{2}+\left(\tilde{e}_{r}^{\frac{1}{2}}\right)^{2}+\left|\tilde{e}_{v}^{\frac{1}{2}}\right|_{\frac{\alpha}{2}}^{2}+\kappa\left|\tilde{e}_{u}^{\frac{1}{2}}\right|_{\alpha}^{2} \leq C \tau^{3} .
$$

By virtue of Lemma 2.3, (4.33) and (4.38), we have

$$
\left\|\tilde{U}^{\frac{1}{2}}\right\|_{\infty} \leq\left\|\tilde{u}^{\frac{1}{2}}\right\|_{\infty}+\left\|\tilde{e}_{u}^{\frac{1}{2}}\right\|_{\infty} \leq\left\|\tilde{u}^{\frac{1}{2}}\right\|_{\infty}+C\left|\tilde{e}_{u}^{\frac{1}{2}}\right|_{\alpha} \leq 1+L \leq M
$$

where $\tau \leq C^{-\frac{2}{3}}$.

2. The second step: estimate $e_{u}^{1}, e_{v}^{1}$ and $e_{r}^{1}$.

Subtract (4.1)-4.3) from 4.14)-4.16) to obtain for $n=0$.

$$
\begin{aligned}
& \delta_{t} e_{u}^{\frac{1}{2}}=\bar{e}_{v}^{\frac{1}{2}}+Q_{1}^{\frac{1}{2}}, \\
& \delta_{t} e_{v}^{\frac{1}{2}}+\kappa(-\Delta)^{\frac{\alpha}{2}} \bar{e}_{u}^{\frac{1}{2}}+\gamma_{1}(-\Delta)^{\frac{\alpha}{2}} \bar{e}_{v}^{\frac{1}{2}}+\gamma_{2} \bar{e}_{v}^{\frac{1}{2}}+G^{\frac{1}{2}}=Q_{2}^{\frac{1}{2}}, \\
& \delta_{t} e_{r}^{\frac{1}{2}}=F^{\frac{1}{2}}+Q_{3}^{\frac{1}{2}},
\end{aligned}
$$

where

$$
\begin{gathered}
G^{\frac{1}{2}}=r^{\frac{1}{2}} \frac{F^{\prime}\left(\tilde{u}^{\frac{1}{2}}\right)}{\sqrt{E\left(\tilde{u}^{\frac{1}{2}}\right)}}-R^{\frac{1}{2}} \frac{F^{\prime}\left(\tilde{U}^{\frac{1}{2}}\right)}{\sqrt{E\left(\tilde{U}^{\frac{1}{2}}\right)}}, \\
F^{\frac{1}{2}}=\frac{1}{2 \sqrt{E\left(\tilde{u}^{\frac{1}{2}}\right)}}\left(F^{\prime}\left(\tilde{u}^{\frac{1}{2}}\right), \delta_{t} u^{\frac{1}{2}}\right)-\frac{1}{2 \sqrt{E\left(\tilde{U}^{\frac{1}{2}}\right)}}\left(F^{\prime}\left(\tilde{U}^{\frac{1}{2}}\right), \delta_{t} U^{\frac{1}{2}}\right) .
\end{gathered}
$$

It is obvious that

$$
\begin{aligned}
& G^{\frac{1}{2}}=r^{\frac{1}{2}}\left(\frac{F^{\prime}\left(\tilde{u}^{\frac{1}{2}}\right)}{\sqrt{E\left(\tilde{u}^{\frac{1}{2}}\right)}}-\frac{F^{\prime}\left(\tilde{U}^{\frac{1}{2}}\right)}{\sqrt{E\left(\tilde{U}^{\frac{1}{2}}\right)}}\right)+\bar{e}_{r}^{\frac{1}{2}} \frac{F^{\prime}\left(\tilde{U}^{\frac{1}{2}}\right)}{\sqrt{E\left(\tilde{U}^{\frac{1}{2}}\right)}}, \\
& F^{\frac{1}{2}}=\left(\frac{1}{2 \sqrt{E\left(\tilde{u}^{\frac{1}{2}}\right)}} F^{\prime}\left(\tilde{u}^{\frac{1}{2}}\right)-\frac{1}{2 \sqrt{E\left(\tilde{U}^{\frac{1}{2}}\right)}} F^{\prime}\left(\tilde{U}^{\frac{1}{2}}\right), \delta_{t} u^{\frac{1}{2}}\right)+\left(\frac{1}{2 \sqrt{E\left(\tilde{U}^{\frac{1}{2}}\right)}} F^{\prime}\left(\tilde{U}^{\frac{1}{2}}\right), \delta_{t} e_{u}^{\frac{1}{2}}\right),
\end{aligned}
$$




$$
\frac{1}{\sqrt{E\left(\tilde{u}^{\frac{1}{2}}\right)}} F^{\prime}\left(\tilde{u}^{\frac{1}{2}}\right)-\frac{1}{\sqrt{E\left(\tilde{U}^{\frac{1}{2}}\right)}} F^{\prime}\left(\tilde{U}^{\frac{1}{2}}\right)=\frac{F^{\prime}\left(\tilde{u}^{\frac{1}{2}}\right)-F^{\prime}\left(\tilde{U}^{\frac{1}{2}}\right)}{\sqrt{E\left(\tilde{u}^{\frac{1}{2}}\right)}}+\frac{F^{\prime}\left(\tilde{U}^{\frac{1}{2}}\right)\left(E\left(\tilde{u}^{\frac{1}{2}}\right)-E\left(\tilde{U}^{\frac{1}{2}}\right)\right)}{\left.\sqrt{E\left(\tilde{U}^{\frac{1}{2}}\right)} \sqrt{E\left(\tilde{u}^{\frac{1}{2}}\right)}\left(\sqrt{E\left(\tilde{u}^{\frac{1}{2}}\right.}\right)+\sqrt{E\left(\tilde{U}^{\frac{1}{2}}\right)}\right)} .
$$

Since $e_{u}^{0}=0, e_{v}^{0}=0$, and $e_{r}^{0}=0$, then taking inner product of $2 \tau e_{u}^{1}$ and $2 \tau e_{v}^{1}$ in (4.40)-(4.41), and multiplying $2 \tau e_{r}^{1}$ on the both sides of (4.42), we have

$$
\begin{aligned}
& 2\left\|e_{u}^{1}\right\|^{2}=\tau\left(e_{v}^{1}, e_{u}^{1}\right)+2 \tau\left(Q_{1}^{\frac{1}{2}}, e_{u}^{1}\right) \\
& 2\left\|e_{v}^{1}\right\|^{2}+\kappa \tau\left((-\Delta)^{\frac{\alpha}{2}} e_{u}^{1}, e_{v}^{1}\right)+\tau \gamma_{1}\left((-\Delta)^{\frac{\alpha}{2}} e_{v}^{1}, e_{v}^{1}\right)+\gamma_{2} \tau\left(e_{v}^{1}, e_{v}^{1}\right)+2 \tau\left(G^{\frac{1}{2}}, e_{v}^{1}\right)=2 \tau\left(Q_{2}^{\frac{1}{2}}, e_{v}^{1}\right)(4 \\
& \left(e_{r}^{1}\right)^{2}=\tau\left(F^{\frac{1}{2}}, e_{r}^{1}\right)+\tau\left(Q_{3}^{\frac{1}{2}}, e_{r}^{1}\right)
\end{aligned}
$$

By using the Cauchy-Schwarz inequality and Young's inequality, we obtain

$$
\begin{aligned}
& \left\|e_{u}^{1}\right\|^{2} \leq C \tau\left\|e_{v}^{1}\right\|^{2}+C \tau\left\|e_{u}^{1}\right\|^{2}+C \tau\left\|Q_{1}^{\frac{1}{2}}\right\|^{2} \\
& \begin{aligned}
2\left\|e_{v}^{1}\right\|^{2}+2 \kappa\left|e_{u}^{1}\right|_{\frac{\alpha}{2}}^{2}+\tau \gamma_{1}\left|e_{v}^{1}\right|_{\frac{\alpha}{2}}^{2} & +\gamma_{2} \tau\left\|e_{v}^{1}\right\|^{2} \leq C \tau\left(\left|\left(G^{\frac{1}{2}}, e_{v}^{1}\right)\right|+\left\|e_{v}^{1}\right\|^{2}+\left\|Q_{2}^{\frac{1}{2}}\right\|^{2}+\left|\left((-\Delta)^{\frac{\alpha}{4}} e_{u}^{1},(-\Delta)^{\frac{\alpha}{4}} Q_{1}^{\frac{1}{2}}\right)\right|\right) \\
& \leq C \tau\left|\left(G^{\frac{1}{2}}, e_{v}^{1}\right)\right|+C \tau\left\|e_{v}^{1}\right\|^{2}+C \tau\left\|Q_{2}^{\frac{1}{2}}\right\|^{2}+C \tau\left|e_{u}^{1}\right|_{\frac{\alpha}{2}}^{2}+C \tau\left|Q_{1}^{\frac{1}{2}}\right|_{\frac{\alpha}{2}}^{2}
\end{aligned} \\
& \left(e_{r}^{1}\right)^{2} \leq C \tau\left|\left(F^{\frac{1}{2}}, e_{r}^{1}\right)\right|+C \tau\left\|e_{r}^{1}\right\|^{2}+C \tau\left\|Q_{3}^{\frac{1}{2}}\right\|^{2}
\end{aligned}
$$

Since $\left|\tilde{U}^{\frac{1}{2}}\right|_{\infty}$ is bounded,

$$
\begin{aligned}
& \left|\left(G^{\frac{1}{2}}, e_{v}^{1}\right)\right| \leq\left(\left|r^{\frac{1}{2}}\right|_{\infty}\left|\tilde{e}_{u}^{\frac{1}{2}}\right|+\left.\left|e_{r}^{\frac{1}{2}}\right|\left|\frac{F^{\prime}\left(\tilde{U}^{\frac{1}{2}}\right)}{\sqrt{E\left(\tilde{U}^{\frac{1}{2}}\right)}}\right|\right|_{\infty}\right)\left|e_{v}^{1}\right| \leq C|| \tilde{e}_{u}^{\frac{1}{2}}\left\|^{2}+C\left(e_{r}^{\frac{1}{2}}\right)^{2}+C\right\| e_{v}^{1} \|^{2}, \\
& \left|\left(F^{\frac{1}{2}}, e_{r}^{1}\right)\right| \leq C\left|e_{r}^{1}\right|\left|\left(\frac{1}{2 \sqrt{E\left(\tilde{u}^{\frac{1}{2}}\right)}} F^{\prime}\left(\tilde{u}^{\frac{1}{2}}\right)-\frac{1}{2 \sqrt{E\left(\tilde{U}^{\frac{1}{2}}\right)}} F^{\prime}\left(\tilde{U}^{\frac{1}{2}}\right), \frac{u^{1}-u^{0}}{\tau}\right)\right|+\left|e_{r}^{1}\right|\left|\left(\frac{1}{2 \sqrt{E\left(\tilde{U}^{\frac{1}{2}}\right)}} F^{\prime}\left(\tilde{U}^{\frac{1}{2}}\right), \delta_{t} e_{u}^{\frac{1}{2}}\right)\right| .
\end{aligned}
$$

where

$$
u^{1}-u^{0}=\tau u_{t}\left(t_{\frac{1}{2}}\right)-\frac{1}{2}\left(\int_{t_{0}}^{t_{1}}\left(t_{0}-s\right)^{2} \frac{\partial^{3} u}{\partial t^{3}}(s) d s+\int_{t_{1}}^{t_{1}}\left(t_{1}-s\right)^{2} \frac{\partial^{3} u}{\partial t^{3}}(s) d s\right) .
$$

From the above equations and (4.40), using Cauchy-Schwarz inequality and Young's inequality, Thus,

$$
\tau\left|\left(F^{\frac{1}{2}}, e_{r}^{1}\right)\right| \leq C \tau\left|u_{t}\left(t_{\frac{1}{2}}\right)\right|_{\infty}\left(\left(e_{r}^{1}\right)^{2}+\left\|\tilde{e}_{u}^{\frac{1}{2}}\right\|^{2}\right)+C \tau^{4} \int_{t_{0}}^{t_{1}}\left\|\frac{\partial^{3} u}{\partial t^{3}}(s)\right\|^{2} d s+C \tau\left(e_{r}^{1}\right)^{2}+C \tau\left\|e_{v}^{1}\right\|^{2}+C \tau\left\|Q_{1}^{\frac{1}{2}}\right\|^{2} .
$$

Combining (4.38) and 4.46)-4.50, we get

$$
\begin{array}{r}
\left\|e_{u}^{1}\right\|^{2}+\left\|e_{v}^{1}\right\|^{2}+\kappa\left|e_{u}^{1}\right|_{\frac{\alpha}{2}}^{2}+\left(e_{r}^{1}\right)^{2} \leq C \tau^{4} \int_{t_{0}}^{t_{1}}\left\|\frac{\partial^{3} u}{\partial t^{3}}(s)\right\|^{2} d s+ \\
C \tau\left(\left\|Q_{2}^{\frac{1}{2}}\right\|^{2}+\left|Q_{1}^{\frac{1}{2}}\right|_{\frac{\alpha}{2}}^{2}+\left\|Q_{3}^{\frac{1}{2}}\right\|^{2}\right)+C \tau\left\|\left.\right|_{e_{u}^{\frac{1}{2}}} ^{\frac{1}{2}}\right\|^{2} \leq C \tau^{4} .
\end{array}
$$


Applying $(-\Delta)^{\frac{\alpha}{2}}$ on the both sides of $(4.40)$, taking the inner product of the resulting equation with $2 \tau(-\Delta)^{\frac{\alpha}{2}} e_{u}^{1}$, one arrives at

$$
2\left|e_{u}^{1}\right|_{\alpha}^{2}=\tau\left((-\Delta)^{\frac{\alpha}{2}} e_{v}^{1},(-\Delta)^{\frac{\alpha}{2}} e_{u}^{1}\right)+2 \tau\left((-\Delta)^{\frac{\alpha}{2}} Q_{1}^{\frac{1}{2}},(-\Delta)^{\frac{\alpha}{2}} e_{u}^{1}\right) .
$$

Taking the inner product of 4.41 with $2 \tau(-\Delta)^{\frac{\alpha}{2}} e_{v}^{1}$ and using 4.52 , we obtain

$$
\begin{array}{r}
2\left|e_{v}^{1}\right|_{\frac{\alpha}{2}}^{2}+2 \kappa\left|e_{u}^{1}\right|_{\alpha}^{2}-2 \kappa \tau\left((-\Delta)^{\frac{\alpha}{2}} Q_{1}^{\frac{1}{2}},(-\Delta)^{\frac{\alpha}{2}} e_{u}^{1}\right)+\gamma_{1}\left|e_{v}^{1}\right|_{\alpha}^{2}+ \\
\gamma_{2} \tau\left|e_{v}^{1}\right|_{\frac{\alpha}{2}}^{2}+2 \tau\left(G^{\frac{1}{2}},(-\Delta)^{\frac{\alpha}{2}} e_{v}^{1}\right)=2 \tau\left(Q_{2}^{\frac{1}{2}},(-\Delta)^{\frac{\alpha}{2}} e_{v}^{1}\right) .
\end{array}
$$

By the Cauchy-Schwarz inequality and Young's inequality, it leads to from the equation (4.54)

$$
2\left|e_{v}^{1}\right|_{\frac{\alpha}{2}}^{2}+2 \kappa\left|e_{u}^{1}\right|_{\alpha}^{2} \leq C \tau\left\|(-\Delta)^{\frac{\alpha}{4}} G^{\frac{1}{2}}\right\|^{2}+C \tau\left|e_{v}^{1}\right|_{\frac{\alpha}{2}}^{2}+C \tau\left\|(-\Delta)^{\frac{\alpha}{4}} Q_{2}^{\frac{1}{2}}\right\|^{2}+C \tau\left\|(-\Delta)^{\frac{\alpha}{2}} Q_{1}^{\frac{1}{2}}\right\|^{2}+C \tau\left|e_{u}^{1}\right|_{\alpha}^{2} .
$$

Meanwhile, we have

$$
\begin{aligned}
& \left\|(-\Delta)^{\frac{\alpha}{4}} G^{\frac{1}{2}}\right\|^{2}=\left\|(-\Delta)^{\frac{\alpha}{4}}\left(r^{\frac{1}{2}}\left(\frac{F^{\prime}\left(\tilde{u}^{\frac{1}{2}}\right)}{\sqrt{E\left(\tilde{u}^{\frac{1}{2}}\right)}}-\frac{F^{\prime}\left(\tilde{U}^{\frac{1}{2}}\right)}{\sqrt{E\left(\tilde{U}^{\frac{1}{2}}\right)}}\right)+\bar{e}_{r}^{\frac{1}{2}} \frac{F^{\prime}\left(\tilde{U}^{\frac{1}{2}}\right)}{\sqrt{E\left(\tilde{U}^{\frac{1}{2}}\right)}}\right)\right\|^{2} \\
& \leq\left|r^{\frac{1}{2}}\right|_{\infty}^{2}\left\|(-\Delta)^{\frac{\alpha}{4}}\left(\frac{F^{\prime}\left(\tilde{u}^{\frac{1}{2}}\right)}{\sqrt{E\left(\tilde{u}^{\frac{1}{2}}\right)}}-\frac{F^{\prime}\left(\tilde{U}^{\frac{1}{2}}\right)}{\sqrt{E\left(\tilde{U}^{\frac{1}{2}}\right)}}\right)\right\|^{2}+\left(e_{r}^{1}\right)^{2}\left\|(-\Delta)^{\frac{\alpha}{4}} \frac{F^{\prime}\left(\tilde{U}^{\frac{1}{2}}\right)}{\sqrt{E\left(\tilde{U}^{\frac{1}{2}}\right)}}\right\|^{2},
\end{aligned}
$$

and by using Lemma 2.2 , we obtain

$$
\begin{aligned}
& \left\|(-\Delta)^{\frac{\alpha}{4}}\left(\frac{F^{\prime}\left(\tilde{u}^{\frac{1}{2}}\right)}{\sqrt{E\left(\tilde{u}^{\frac{1}{2}}\right)}}-\frac{F^{\prime}\left(\tilde{U}^{\frac{1}{2}}\right)}{\sqrt{E\left(\tilde{U}^{\frac{1}{2}}\right)}}\right)\right\|^{2} \leq\left\|(-\Delta)^{\frac{\alpha}{4}} \frac{F^{\prime}\left(\tilde{U}^{\frac{1}{2}}\right)\left(E\left(\tilde{u}^{\frac{1}{2}}\right)-E\left(\tilde{U}^{\frac{1}{2}}\right)\right)}{\sqrt{E\left(\tilde{U}^{\frac{1}{2}}\right)} \sqrt{E\left(\tilde{u}^{\frac{1}{2}}\right)}\left(\sqrt{E\left(\tilde{u}^{\frac{1}{2}}\right)}+\sqrt{E\left(\tilde{U}^{\frac{1}{2}}\right)}\right)}\right\|^{2} \\
& +\left\|(-\Delta)^{\frac{\alpha}{4}} \frac{F^{\prime}\left(\tilde{u}^{\frac{1}{2}}\right)-F^{\prime}\left(\tilde{U}^{\frac{1}{2}}\right)}{\sqrt{E\left(\tilde{u}^{\frac{1}{2}}\right)}}\right\|^{2} \leq\left\|(-\Delta)^{\frac{\alpha}{4}} \frac{\partial_{u} F^{\prime}(\tilde{\xi})\left(\tilde{u}^{\frac{1}{2}}-\tilde{U}^{\frac{1}{2}}\right)}{\sqrt{E\left(\tilde{u}^{\frac{1}{2}}\right)}}\right\|^{2}+C\left|E\left(\tilde{u}^{\frac{1}{2}}\right)-E\left(\tilde{U}^{\frac{1}{2}}\right)\right|^{2}\left\|(-\Delta)^{\frac{\alpha}{4}} F^{\prime}\left(\tilde{U}^{\frac{1}{2}}\right)\right\|^{2}
\end{aligned}
$$

$\leq C\left|\tilde{e}_{u}^{\frac{1}{2}}\right|_{\frac{\alpha}{2}}^{2}$

Then substituting (4.56) into 4.55) and using Lemma 2.2, it follows from (4.54) that

$$
\left\|(-\Delta)^{\frac{\alpha}{4}} G^{\frac{1}{2}}\right\|^{2} \leq C\left|\tilde{e}_{u}^{\frac{1}{2}}\right|_{\frac{\alpha}{2}}^{2}+C\left(e_{r}^{1}\right)^{2} .
$$

Combining (4.51), 4.54) and 4.57), we arrive at

$$
\left|e_{v}^{1}\right|_{\frac{\alpha}{2}}^{2}+\kappa\left|e_{u}^{1}\right|_{\alpha}^{2}+\left\|e_{u}^{1}\right\|^{2}+\left\|e_{v}^{1}\right\|^{2}+\kappa\left|e_{u}^{1}\right|_{\frac{\alpha}{2}}^{2}+\left(e_{r}^{1}\right)^{2} \leq C \tau^{4} .
$$

By virtue of Lemma 2.3 and (4.58), we have

$$
\left\|U^{1}\right\|_{\infty} \leq\left\|u^{1}\right\|_{\infty}+\left\|e_{u}^{1}\right\|_{\infty} \leq\left\|u^{1}\right\|_{\infty}+C\left|e_{u}^{1}\right|_{\alpha} \leq 1+L \leq M,
$$


where $\tau \leq C^{-\frac{1}{2}}$.

3. The third step : we intend to use the mathematical induction method to estimate (4.12) holds for $1 \leq n \leq K$.

First, we assume that (4.12) holds for $n \leq k$ and we intend to prove that it also holds for $n=k+1$. Similar to 4.59$]$, we obtain

$$
\left\|U^{n}\right\|_{\infty} \leq M, 1 \leq n \leq k .
$$

Subtract (4.1)-(4.3) from (4.14)-(4.16) to obtain the following error equations.

$$
\begin{aligned}
& \delta_{t} e_{u}^{k+\frac{1}{2}}=\bar{e}_{v}^{k+\frac{1}{2}}+Q_{1}^{k+\frac{1}{2}}, \\
& \delta_{t} e_{v}^{k+\frac{1}{2}}+\kappa(-\Delta)^{\frac{\alpha}{2}} e_{u}^{k+\frac{1}{2}}+\gamma_{1}(-\Delta)^{\frac{\alpha}{2}} e_{v}^{k+\frac{1}{2}}+\gamma_{2} \bar{e}_{v}^{k+\frac{1}{2}}+G^{k+\frac{1}{2}}=Q_{2}^{k+\frac{1}{2}}, \\
& \delta_{t} e_{r}^{k+\frac{1}{2}}=F^{k+\frac{1}{2}}+Q_{3}^{k+\frac{1}{2}} .
\end{aligned}
$$

Taking the inner product of $2 \tau \bar{e}_{u}^{k+\frac{1}{2}}$ and $2 \tau \bar{e}_{v}^{k+\frac{1}{2}}$ in (4.61)-4.62) respectively, as well as multiplying $2 \tau \bar{e}_{r}^{k+\frac{1}{2}}$ on the both sides of 4.63 , we have

$$
\begin{aligned}
& \left\|e_{u}^{k+1}\right\|^{2}-\left\|e_{u}^{k}\right\|^{2}=2 \tau\left(\bar{e}_{v}^{k+\frac{1}{2}}, \bar{e}_{u}^{k+\frac{1}{2}}\right)+2 \tau\left(Q_{1}^{k+\frac{1}{2}}, \bar{e}_{u}^{k+\frac{1}{2}}\right), \\
& \left\|e_{v}^{k+1}\right\|^{2}-\left\|e_{v}^{k}\right\|^{2}+\kappa\left(\left|e_{u}^{k+1}\right| \frac{\alpha}{2}-\left|e_{u}^{k}\right|_{\frac{\alpha}{2}}^{2}\right)+2 \tau \gamma_{1}\left|\bar{e}_{v}^{k+\frac{1}{2}}\right|_{\frac{\alpha}{2}}^{2}+2 \gamma_{2} \tau\left\|\left.\right|_{v} ^{k+\frac{1}{2}}\right\|^{2} \\
& =2 \tau\left(Q_{2}^{k+\frac{1}{2}}, \bar{e}_{v}^{k+\frac{1}{2}}\right)-2 \tau\left(G^{k+\frac{1}{2}}, \bar{e}_{v}^{k+\frac{1}{2}}\right)+2 \tau \kappa\left(Q_{1}^{k+\frac{1}{2}},(-\Delta)^{\alpha / 2} \bar{e}_{u}^{k+\frac{1}{2}}\right), \\
& \left(e_{r}^{k+1}\right)^{2}-\left(e_{r}^{k}\right)^{2}=2 \tau\left(F^{k+\frac{1}{2}}, \bar{e}_{r}^{k+\frac{1}{2}}\right)+2 \tau\left(Q_{3}^{k+\frac{1}{2}}, \bar{e}_{r}^{k+\frac{1}{2}}\right),
\end{aligned}
$$

where

$$
G^{k+\frac{1}{2}}=r^{k+\frac{1}{2}}\left(\frac{F^{\prime}\left(\tilde{u}^{k+\frac{1}{2}}\right)}{\sqrt{E\left(\tilde{u}^{k+\frac{1}{2}}\right)}}-\frac{F^{\prime}\left(\tilde{U}^{k+\frac{1}{2}}\right)}{\sqrt{E\left(\tilde{U}^{k+\frac{1}{2}}\right)}}\right)+\bar{e}_{r}^{k+\frac{1}{2}} \frac{F^{\prime}\left(\tilde{U}^{k+\frac{1}{2}}\right)}{\sqrt{E\left(\tilde{U}^{k+\frac{1}{2}}\right)}},
$$

and

$$
F^{k+\frac{1}{2}}=\left(\frac{1}{2 \sqrt{E\left(\tilde{u}^{k+\frac{1}{2}}\right)}} F^{\prime}\left(\tilde{u}^{k+\frac{1}{2}}\right)-\frac{1}{2 \sqrt{E\left(\tilde{U}^{k+\frac{1}{2}}\right)}} F^{\prime}\left(\tilde{U}^{k+\frac{1}{2}}\right), \delta_{t} u^{k+\frac{1}{2}}\right)+\left(\frac{1}{2 \sqrt{E\left(\tilde{U}^{k+\frac{1}{2}}\right)}} F^{\prime}\left(\tilde{U}^{k+\frac{1}{2}}\right), \delta_{t} e_{u}^{k+\frac{1}{2}}\right),
$$

where

$$
\begin{aligned}
& \frac{1}{\sqrt{E\left(\tilde{u}^{k+\frac{1}{2}}\right)}} F^{\prime}\left(\tilde{u}^{k+\frac{1}{2}}\right)-\frac{1}{\sqrt{E\left(\tilde{U}^{k+\frac{1}{2}}\right)}} F^{\prime}\left(\tilde{U}^{k+\frac{1}{2}}\right)= \\
& \frac{F^{\prime}\left(\tilde{u}^{k+\frac{1}{2}}\right)-F^{\prime}\left(\tilde{U}^{k+\frac{1}{2}}\right)}{\sqrt{E\left(\tilde{u}^{k+\frac{1}{2}}\right)}}+\frac{F^{\prime}\left(\tilde{U}^{k+\frac{1}{2}}\right)\left(E\left(\tilde{u}^{k+\frac{1}{2}}\right)-E\left(\tilde{U}^{k+\frac{1}{2}}\right)\right)}{\left.\sqrt{E\left(\tilde{U}^{k+\frac{1}{2}}\right)} \sqrt{E\left(\tilde{u}^{k+\frac{1}{2}}\right)}\left(\sqrt{E\left(\tilde{u}^{k+\frac{1}{2}}\right.}\right)+\sqrt{E\left(\tilde{U}^{k+\frac{1}{2}}\right)}\right)} \\
& u^{k+1}-u^{k}=\tau u_{t}\left(t_{k+\frac{1}{2}}\right)-\frac{1}{2}\left(\int_{t_{k}}^{t_{k+\frac{1}{2}}}\left(t_{k}-s\right)^{2} \frac{\partial^{3} u}{\partial t^{3}}(s) d s+\int_{t_{k+1}}^{t_{k+\frac{1}{2}}}\left(t_{k+1}-s\right)^{2} \frac{\partial^{3} u}{\partial t^{3}}(s) d s\right) .
\end{aligned}
$$


Similar to the proof for (4.49) and (4.50), we have the following estimates

$$
\begin{gathered}
\left|\left(G^{k+\frac{1}{2}}, \bar{e}_{v}^{k+\frac{1}{2}}\right)\right| \leq\left(\left|\bar{r}^{k+\frac{1}{2}}\right|_{\infty}\left|\tilde{e}_{u}^{k+\frac{1}{2}}\right|+\left|\bar{e}_{r}^{k+\frac{1}{2}}\right|\left\|\frac{F^{\prime}\left(\tilde{U}^{k+\frac{1}{2}}\right)}{\sqrt{E\left(\tilde{U}^{k+\frac{1}{2}}\right)}}\right\|_{\infty}\right)\left|\bar{e}_{v}^{k+\frac{1}{2}}\right| \\
\leq C\left(\left\|e_{u}^{k}\right\|^{2}+\left\|e_{u}^{k-1}\right\|^{2}+\left\|e_{r}^{k+1}\right\|^{2}+\left\|e_{v}^{k+1}\right\|^{2}+\left\|e_{r}^{k}\right\|^{2}+\left\|e_{v}^{k}\right\|^{2}\right), \\
\tau\left|\left(F^{k+\frac{1}{2}}, \bar{e}_{r}^{k+\frac{1}{2}}\right)\right| \leq C \tau\left|u_{t}\left(t_{k+\frac{1}{2}}\right)\right|_{\infty}\left(\left(e_{r}^{k+1}\right)^{2}+\left(e_{r}^{k}\right)^{2}+\left\|e_{u}^{k}\right\|^{2}+\left\|e_{u}^{k-1}\right\|^{2}\right)+ \\
C \tau^{4} \int_{t_{k}}^{t_{k+1}}\left\|\frac{\partial^{3} u}{\partial t^{3}}(s)\right\|^{2} d s+C \tau\left(\left\|e_{v}^{k+1}\right\|^{2}+\left\|e_{v}^{k}\right\|^{2}+\left\|Q_{1}^{k+\frac{1}{2}}\right\|^{2}\right) .
\end{gathered}
$$

Combining 4.69-(4.70), using Cauchy-Schwarz inequality and Young's inequality, we get

$$
\begin{aligned}
&\left\|e_{u}^{k+1}\right\|^{2}-\left\|e_{u}^{k}\right\|^{2}+\left\|e_{v}^{k+1}\right\|^{2}-\left\|e_{v}^{k}\right\|^{2}+\kappa\left(\left|e_{u}^{k+1}\right|_{\frac{\alpha}{2}}^{2}-\left|e_{u}^{k}\right|_{\frac{\alpha}{2}}^{2}\right)+\left(e_{r}^{k+1}\right)^{2}-\left(e_{r}^{k}\right)^{2} \\
& \leq C \tau\left(\left\|e_{v}^{k+1}\right\|^{2}+\left\|e_{v}^{k}\right\|^{2}+\left\|e_{u}^{k}\right\|^{2}+\left\|e_{u}^{k-1}\right\|^{2}+\left(e_{r}^{k+1}\right)^{2}+\left(e_{r}^{k}\right)^{2}+\left|e_{u}^{k+1}\right|_{\frac{\alpha}{2}}^{2}+\left|e_{u}^{k}\right|_{\frac{\alpha}{2}}^{2}\right)+ \\
& C \tau\left(\left\|Q_{1}^{k+\frac{1}{2}}\right\|^{2}+\left|Q_{1}^{k+\frac{1}{2}}\right|_{\frac{\alpha}{2}}^{2}+\left\|Q_{2}^{k+\frac{1}{2}}\right\|^{2}+\left\|Q_{3}^{k+\frac{1}{2}}\right\|^{2}\right)+C \tau^{4} \int_{t_{k}}^{t_{k+1}}\left\|\frac{\partial^{3} u}{\partial t^{3}}(s)\right\|^{2} d s
\end{aligned}
$$

Applying $(-\Delta)^{\frac{\alpha}{2}}$ on the both sides of (4.61), and then taking the inner product of the resulting equation with $\tau(-\Delta)^{\frac{\alpha}{2}} \bar{e}_{u}^{k+\frac{1}{2}}$, one arrives at

$$
\left|e_{u}^{k+1}\right|_{\alpha}^{2}-\left|e_{u}^{k}\right|_{\alpha}^{2}=\tau\left((-\Delta)^{\frac{\alpha}{2}} \bar{e}_{v}^{k+\frac{1}{2}},(-\Delta)^{\frac{\alpha}{2}} \bar{e}_{u}^{k+\frac{1}{2}}\right)+\tau\left((-\Delta)^{\frac{\alpha}{2}} Q_{1}^{k+\frac{1}{2}},(-\Delta)^{\frac{\alpha}{2}} \bar{e}_{u}^{k+\frac{1}{2}}\right),
$$

and taking the inner product of (4.62) with $2 \tau(-\Delta)^{\frac{\alpha}{2}} \bar{e}_{v}^{k+\frac{1}{2}}$, we get

$$
\begin{aligned}
& \left|e_{v}^{k+1}\right|_{\frac{\alpha}{2}}^{2}-\left|e_{v}^{k}\right|_{\frac{\alpha}{2}}^{2}+\kappa\left|e_{u}^{k+1}\right|_{\alpha}^{2}-\kappa\left|e_{u}^{k}\right|_{\alpha}^{2}+\tau \gamma_{1}\left|\bar{e}_{v}^{k+\frac{1}{2}}\right|_{\alpha}^{2}+\gamma_{2} \tau\left|\bar{e}_{v}^{k+\frac{1}{2}}\right|_{\frac{\alpha}{2}}^{2} \\
& =-\tau\left(G^{k+\frac{1}{2}},(-\Delta)^{\frac{\alpha}{2}} \bar{e}_{v}^{k+\frac{1}{2}}\right)+\tau\left(Q_{2}^{k+\frac{1}{2}},(-\Delta)^{\frac{\alpha}{2}} e_{v}^{k+\frac{1}{2}}\right)+\kappa \tau\left((-\Delta)^{\frac{\alpha}{2}} Q_{1}^{k+\frac{1}{2}},(-\Delta)^{\frac{\alpha}{2}} \bar{e}_{u}^{k+\frac{1}{2}}\right) .
\end{aligned}
$$

Using Lemmas 2.1 and 2.2, one obtains

$$
\left\|(-\Delta)^{\frac{\alpha}{4}} G^{k+\frac{1}{2}}\right\|^{2} \leq C \tau\left(\left|e_{u}^{k}\right|_{\frac{\alpha}{2}}^{2}+\left|e_{u}^{k-1}\right|_{\frac{\alpha}{2}}^{2}+\left(e_{r}^{k+1}\right)^{2}+\left(e_{r}^{k}\right)^{2}\right),
$$

then from 4.73 - 4.74 ), we get

$$
\begin{aligned}
& \left|e_{v}^{k+1}\right|_{\frac{\alpha}{2}}^{2}-\left|e_{v}^{k}\right|_{\frac{\alpha}{2}}^{2}+\kappa\left|e_{u}^{k+1}\right|_{\alpha}^{2}-\kappa\left|e_{u}^{k}\right|_{\alpha}^{2} \leq C \tau\left(\left|e_{v}^{k+1}\right|_{\alpha}^{2}+\left|e_{v}^{k}\right|_{\alpha}^{2}+\left|e_{u}^{k+1}\right|_{\alpha}^{2}+\left|e_{u}^{k}\right|_{\alpha}^{2}+\right. \\
& \left.\left|e_{u}^{k}\right|_{\alpha / 2}^{2}+\left|e_{u}^{k-1}\right|_{\alpha / 2}^{2}+\left(e_{r}^{k+1}\right)^{2}+\left(e_{r}^{k}\right)^{2}\right)+\left\|(-\Delta)^{\frac{\alpha}{2}} Q_{1}^{k+\frac{1}{2}}\right\|^{2}+\left\|(-\Delta)^{\frac{\alpha}{4}} Q_{2}^{k+\frac{1}{2}}\right\|^{2} .
\end{aligned}
$$

Combining (4.71) and (4.75), summing from $n=1$ to $n=k$, and then using Gronwall inequality, we get

$$
\left\|e_{u}^{k+1}\right\|^{2}+\left\|e_{v}^{k+1}\right\|^{2}+\kappa\left|e_{v}^{k+1}\right|_{\frac{\alpha}{2}}^{2}+\kappa\left|e_{u}^{k+1}\right|_{\frac{\alpha}{2}}^{2}+\kappa\left|e_{u}^{k+1}\right|_{\alpha}^{2}+\left(e_{r}^{k+1}\right)^{2} \leq C \tau^{4},
$$

which implies $\left|e_{u}^{k+1}\right|_{\alpha} \leq C \tau^{2}$. Moreover, we arrive at

$$
\left\|U^{k+1}\right\|_{\infty} \leq\left\|u^{k+1}\right\|_{\infty}+\left\|e_{u}^{k+1}\right\|_{\infty} \leq L+\left|e_{u}^{k+1}\right|_{\alpha} \leq M .
$$

(4.76) shows that 4.12) holds for $n=k+1$. We have completed the mathematical induction and thus the proof is completed. 


\section{Spatial error analysis}

In this section, we give the $\tau$-independent convergence results for the discrete full scheme. It implies that we do not need to assume the global Lipshitz condition. Similar to the technique in [40], from Lemma 2.3 and Lemma 2.5, we have $\left\|P_{N} w\right\|_{\infty} \leq C\|w\|_{\alpha}$. Then we have the boundedness of $P_{N} U^{n}$ in $L_{\infty}$ norm, and denote $M_{2}=\max _{0 \leq n \leq K}\left\{P_{N} U^{n}\right\}+1$. For convenience, we denote

$$
\begin{aligned}
& \bar{e}_{N, u}^{n}=U^{n}-u_{N}^{n}=U^{n}-P_{N} U^{n}+P_{N} U^{n}-u_{N}^{n}=\rho_{u}^{n}+\theta_{u}^{n}, \\
& \bar{e}_{N, v}^{n}=V^{n}-v_{N}^{n}=V^{n}-P_{N} V^{n}+P_{N} V^{n}-v_{N}^{n}=\rho_{v}^{n}+\theta_{v}^{n} .
\end{aligned}
$$

Combining the time-discrete system (4.1)-(4.3) and (3.9)-(3.15), we directly get the following error equations

$$
\begin{aligned}
& \left(\delta_{t} \theta_{u}^{n+\frac{1}{2}}, \psi\right)=\left(\bar{\theta}_{v}^{n+\frac{1}{2}}, \psi\right), \\
& \left(\delta_{t} \theta_{v}^{n+\frac{1}{2}}, \varphi\right)+\kappa\left((-\Delta)^{\frac{\alpha}{2}} \bar{\theta}_{u}^{n+\frac{1}{2}}, \varphi\right)+\gamma_{1}\left((-\Delta)^{\frac{\alpha}{2}} \bar{\theta}_{v}^{n+\frac{1}{2}}, \varphi\right)+\gamma_{2}\left(\bar{\theta}_{v}^{n+\frac{1}{2}}, \varphi\right)+\left(R_{1}^{n+\frac{1}{2}}, \varphi\right)=0,
\end{aligned}
$$

where $R_{1}^{n+\frac{1}{2}}=\bar{R}^{n+\frac{1}{2}}\left(\frac{F^{\prime}\left(\tilde{u}_{N}^{n+\frac{1}{2}}\right)}{\sqrt{E\left(\tilde{u}_{N}^{n+\frac{1}{2}}\right)}}-\frac{F^{\prime}\left(\tilde{U}^{n+\frac{1}{2}}\right)}{\sqrt{E\left(\tilde{U}^{n+\frac{1}{2}}\right)}}\right)$.

Following from (3.12), we have $\theta_{u}^{0}=0$ and $\theta_{v}^{0}=0$. Moreover, for the first step, we have

$$
\begin{aligned}
& \left(\frac{\tilde{\theta}_{u}^{\frac{1}{2}}}{\tau / 2}, \psi\right)=\left(\tilde{\theta}_{v}^{\frac{1}{2}}, \psi\right), \\
& \left(\frac{\tilde{\theta}_{v}^{\frac{1}{2}}}{\tau / 2}, \varphi\right)+\kappa\left((-\Delta)^{\frac{\alpha}{2}} \tilde{\theta}_{u}^{\frac{1}{2}}, \varphi\right)+\gamma_{1}\left((-\Delta)^{\frac{\alpha}{2}} \tilde{\theta}_{v}^{\frac{1}{2}}, \varphi\right)+\gamma_{2}\left(\tilde{\theta}_{v}^{\frac{1}{2}}, \varphi\right)+\left(\tilde{R}_{1}^{\frac{1}{2}}, \varphi\right)=0,
\end{aligned}
$$

where $\tilde{R}_{1}^{\frac{1}{2}}=\tilde{R}^{\frac{1}{2}}\left(\frac{F^{\prime}\left(u_{N}^{0}\right)}{\sqrt{E\left(u_{N}^{0}\right)}}-\frac{F^{\prime}\left(U^{0}\right)}{\sqrt{E\left(U^{0}\right)}}\right)$.

Theorem 5.1. Suppose that (3.1)-(3.4) have unique solutions satisfying (3.19) and $u_{N}^{n}$, $v_{N}^{n}$ are the solutions of (3.9)-(3.11), $0 \leq n \leq K$. Then there exist two positive constants $\tau^{*}$ and $N^{*}$, such that when $\tau \leq \tau^{*}$ and $N \geq N^{*}$, we have

$$
\begin{aligned}
& \left\|\theta_{u}^{n}\right\|^{2}+\left\|\theta_{v}^{n}\right\|^{2}+\kappa\left|\theta_{u}^{n}\right|_{\frac{\alpha}{2}}^{2} \leq C N^{-2 \alpha}, 0 \leq n \leq K, \\
& \left\|u_{N}^{n}\right\|_{\infty} \leq M_{2} .
\end{aligned}
$$

Proof. 1. The first step: estimate $\tilde{\theta}_{u}^{\frac{1}{2}}$ and $\tilde{\theta}_{v}^{\frac{1}{2}}$.

Letting $\psi=\tilde{\theta}_{u}^{\frac{1}{2}}$ and $\varphi=\tilde{\theta}_{v}^{\frac{1}{2}}$ in $(5.4)-(5.5)$, we can get

$$
\begin{aligned}
& \left\|\tilde{\theta}_{u}^{\frac{1}{2}}\right\|^{2}=\frac{\tau}{2}\left(\tilde{\theta}_{u}^{\frac{1}{2}}, \tilde{\theta}_{v}^{\frac{1}{2}}\right), \\
& \left\|\tilde{\theta}_{v}^{\frac{1}{2}}\right\|^{2}+\frac{\tau}{2} \kappa\left((-\Delta)^{\frac{\alpha}{2}} \tilde{\theta}_{u}^{\frac{1}{2}}, \tilde{\theta}_{v}^{\frac{1}{2}}\right)+\frac{\tau}{2} \gamma_{1}\left|\tilde{\theta}_{v}^{\frac{1}{2}}\right|_{\frac{\alpha}{2}}^{2}+\frac{\tau}{2} \gamma_{2}\left\|\tilde{\theta}_{v}^{\frac{1}{2}}\right\|^{2}=-\frac{\tau}{2}\left(\tilde{R}_{1}^{\frac{1}{2}}, \tilde{\theta}_{v}^{\frac{1}{2}}\right) .
\end{aligned}
$$


Setting $\psi=(-\Delta)^{\frac{\alpha}{2}} \tilde{\theta}_{u}^{\frac{1}{2}}$ in $(5.8)$, the (5.9) can be rewritten as

$$
\left\|\tilde{\theta}_{v}^{\frac{1}{2}}\right\|^{2}+\kappa\left|\tilde{\theta}_{u}^{\frac{1}{2}}\right|_{\frac{\alpha}{2}}^{2}+\frac{\tau}{2} \gamma_{1}\left|\tilde{\theta}_{v}^{\frac{1}{2}}\right|_{\frac{\alpha}{2}}^{2}+\frac{\tau}{2} \gamma_{2}\left\|\tilde{\theta}_{v}^{\frac{1}{2}}\right\|^{2}=-\frac{\tau}{2}\left(\tilde{R}_{1}^{\frac{1}{2}}, \tilde{\theta}_{v}^{\frac{1}{2}}\right)
$$

meanwhile $\tilde{R}_{1}^{\frac{1}{2}}$ also could be represented by

$$
\tilde{R}_{1}^{\frac{1}{2}}=\tilde{R}^{\frac{1}{2}}\left(\frac{F^{\prime}\left(u_{N}^{0}\right)-F^{\prime}\left(U^{0}\right)}{\sqrt{E\left(u_{N}^{0}\right)}}+\frac{F^{\prime}\left(U^{0}\right)\left(E\left(u_{N}^{0}\right)-E\left(U^{0}\right)\right)}{\sqrt{E\left(u_{N}^{0}\right)} \sqrt{E\left(U^{0}\right)}\left(\sqrt{E\left(u_{N}^{0}\right)}+\sqrt{E\left(U^{0}\right)}\right)}\right) .
$$

From Theorem 4.2 and $\left(\tilde{e}_{r}^{\frac{1}{2}}\right)^{2} \leq C \tau^{3}$, thus $\left|\tilde{R}^{\frac{1}{2}}\right|$ is bounded. We denote

$$
\hat{K}=\max \left\{\left|\tilde{r}^{\frac{1}{2}}\right|,\left|\tilde{R}^{\frac{1}{2}}\right|\right\}+1,
$$

Combining (5.8)-(5.12), we can get

$$
\left\|\tilde{\theta}_{u}^{\frac{1}{2}}\right\|^{2}+\left\|\tilde{\theta}_{v}^{\frac{1}{2}}\right\|^{2}+\kappa\left|\tilde{\theta}_{u}^{\frac{1}{2}}\right|_{\frac{\alpha}{2}}^{2} \leq C \tau^{2}\left\|\tilde{\theta}_{v}^{\frac{1}{2}}\right\|^{2}+C\left\|\rho_{u}^{0}\right\|^{2}+C \tau\left\|\tilde{\theta}_{u}^{\frac{1}{2}}\right\|^{2}+C \tau\left\|\tilde{\theta}_{v}^{\frac{1}{2}}\right\|^{2} .
$$

Thus, by Lemma 2.5, we can have

$$
\left\|\tilde{\theta}_{u}^{\frac{1}{2}}\right\|^{2}+\left\|\tilde{\theta}_{v}^{\frac{1}{2}}\right\|^{2}+\kappa\left|\tilde{\theta}_{u}^{\frac{1}{2}}\right|_{\frac{\alpha}{2}}^{2} \leq N^{-2 \alpha}
$$

From Lemma 2.4, we have

$$
\left\|\tilde{u}_{N}^{\frac{1}{2}}\right\|_{\infty} \leq\left\|P_{N} \tilde{U}^{\frac{1}{2}}\right\|_{\infty}+\left\|\tilde{\theta}_{u}^{\frac{1}{2}}\right\|_{\infty} \leq\left\|P_{N} \tilde{U}^{\frac{1}{2}}\right\|_{\infty}+C N^{1-\alpha} \leq M_{2},
$$

where $\tau \leq \tau^{*}$ and $N \geq C^{\frac{1}{\alpha-1}}$.

2. The second step: estimate $\theta_{u}^{1}$ and $\theta_{v}^{1}$.

Letting $\psi=\theta_{u}^{1}$ and $\varphi=\theta_{v}^{1}$ in (5.2)-(5.3), then it leads to

$$
\begin{aligned}
& \left\|\theta_{u}^{1}\right\|^{2}=\frac{\tau}{2}\left(\theta_{v}^{1}, \theta_{u}^{1}\right), \\
& \left\|\theta_{v}^{1}\right\|^{2}+\kappa\left|\theta_{u}^{1}\right|_{\frac{\alpha}{2}}^{2}+\frac{\tau}{2} \gamma_{1}\left|\theta_{v}^{1}\right|_{\frac{\alpha}{2}}^{2}+\gamma_{2} \frac{\tau}{2}\left\|\theta_{v}^{1}\right\|^{2}=\frac{\tau}{2}\left(R_{1}^{\frac{1}{2}}, \theta_{v}^{1}\right) .
\end{aligned}
$$

Using

$$
R_{1}^{\frac{1}{2}}=\bar{R}^{\frac{1}{2}}\left(\frac{F^{\prime}\left(\tilde{u}_{N}^{\frac{1}{2}}\right)-F^{\prime}\left(\tilde{U}^{\frac{1}{2}}\right)}{\sqrt{E\left(\tilde{u}_{N}^{\frac{1}{2}}\right)}}+\frac{F^{\prime}\left(\tilde{U}^{\frac{1}{2}}\right)\left(E\left(\tilde{u}_{N}^{\frac{1}{2}}\right)-E\left(\tilde{U}^{\frac{1}{2}}\right)\right)}{\left.\sqrt{E\left(\tilde{U}^{\frac{1}{2}}\right)} \sqrt{E\left(\tilde{u}_{N}^{\frac{1}{2}}\right)}\left(\sqrt{E\left(\tilde{u}_{N}^{\frac{1}{2}}\right.}\right)+\sqrt{E\left(\tilde{U}^{\frac{1}{2}}\right)}\right)},\right.
$$

and from Theorem 4.2, we get the boundedness of $\left|\bar{R}^{\frac{1}{2}}\right|$. We denote $\hat{K}_{1}=\max \left\{\left|\bar{R}^{\frac{1}{2}}\right|,\left|\bar{r}^{\frac{1}{2}}\right|\right\}+1$, and by virtue of Lemma 2.5 , we have

$$
\left\|\theta_{u}^{1}\right\|^{2}+\left\|\theta_{v}^{1}\right\|^{2}+\kappa\left|\theta_{u}^{1}\right|_{\frac{\alpha}{2}}^{2} \leq C N^{-2 \alpha}
$$


Moreover, when $\tau \leq \tau^{*}$ and $N \geq C^{\frac{1}{\alpha-1}}$,

$$
\left\|u_{N}^{1}\right\|_{\infty} \leq\left\|P_{N} U^{1}\right\|_{\infty}+\left\|\theta_{u}^{1}\right\|_{\infty} \leq\left\|P_{N} U^{1}\right\|_{\infty}+C N^{1-\alpha} \leq M_{2} .
$$

3. The third step. Suppose that (5.6) holds for $0 \leq n \leq k$ and discuss that the case for $n=k+1$ holds.

We use the mathematical induction method and assume

$$
\left\|u_{N}^{n}\right\|_{\infty} \leq M_{2}, 0 \leq n \leq k,
$$

and denote $\hat{K}_{2}=\max \left\{\left|\tilde{r}^{n+\frac{1}{2}}\right|,\left|\tilde{R}^{n+\frac{1}{2}}\right|(n=0, \ldots, k)\right\}+1$. Next, we intend to prove that 5.6$)$ holds for $n=k+1$. Letting $\psi=\tau \bar{\theta}_{u}^{k+\frac{1}{2}}$ and $\varphi=\tau \bar{\theta}_{v}^{k+\frac{1}{2}}$ in (5.2)-(5.3), respectively, we get

$$
\begin{aligned}
& \left\|\theta_{u}^{k+1}\right\|^{2}-\left\|\theta_{u}^{k}\right\|^{2}=\tau\left(\bar{\theta}_{v}^{k+\frac{1}{2}}, \bar{\theta}_{u}^{k+\frac{1}{2}}\right), \\
& \left\|\theta_{v}^{k+1}\right\|^{2}-\left\|\theta_{v}^{k}\right\|^{2}+\kappa\left(\left|\theta_{u}^{k+1}\right|_{\frac{\alpha}{2}}^{2}-\left|\theta_{u}^{k}\right|_{\frac{\alpha}{2}}^{2}\right)+\tau \gamma_{1}\left|\bar{\theta}_{v}^{k+\frac{1}{2}}\right|_{\frac{\alpha}{2}}^{2}+\gamma_{2} \tau\left\|\left.\right|_{v} ^{k+\frac{1}{2}}\right\|^{2}=-\tau\left(R_{1}^{k+\frac{1}{2}}, \bar{\theta}_{v}^{k+\frac{1}{2}}\right) .
\end{aligned}
$$

By using Cauchy-Schwarz inequality and Young's inequality, we have

$$
\begin{aligned}
& \left|\left(R_{1}^{k+\frac{1}{2}}, \bar{\theta}_{v}^{k+\frac{1}{2}}\right)\right| \leq\left\|\theta_{u}^{k}\right\|^{2}+\left\|\theta_{u}^{k-1}\right\|^{2}+\left\|\theta_{v}^{k}\right\|^{2}+\left\|\theta_{v}^{k+1}\right\|^{2}+\left\|\rho_{u}^{k}\right\|^{2}+\left\|\rho_{u}^{k-1}\right\|^{2}, \\
& \left|\left(\bar{\theta}_{v}^{k+\frac{1}{2}}, \bar{\theta}_{u}^{k+\frac{1}{2}}\right)\right| \leq \frac{1}{4}\left(\left\|\theta_{u}^{k}\right\|^{2}+\left\|\theta_{u}^{k+1}\right\|^{2}+\left\|\theta_{v}^{k}\right\|^{2}+\left\|\theta_{v}^{k+1}\right\|^{2}\right) .
\end{aligned}
$$

Therefore, we get

$$
\begin{aligned}
& \left\|\theta_{u}^{k+1}\right\|^{2}-\left\|\theta_{u}^{k}\right\|^{2}+\left\|\theta_{v}^{k+1}\right\|^{2}-\left\|\theta_{v}^{k}\right\|^{2}+\kappa\left(\left|\theta_{u}^{k+1}\right|_{\frac{\alpha}{2}}^{2}-\left|\theta_{u}^{k}\right|_{\frac{\alpha}{2}}^{2}\right) \leq \\
& C \tau\left(\left\|\theta_{u}^{k}\right\|^{2}+\left\|\theta_{u}^{k+1}\right\|^{2}+\left\|\theta_{v}^{k}\right\|^{2}+\left\|\theta_{v}^{k+1}\right\|^{2}+\left\|\rho_{u}^{k}\right\|^{2}+\left\|\rho_{u}^{k-1}\right\|^{2}+\left\|\theta_{u}^{k-1}\right\|^{2}\right) .
\end{aligned}
$$

Summing from $n=1$ to $n=k$ and using the discrete Gronwall's inequality and Lemma 2.5, (5.25) leads to

$$
\left\|\theta_{u}^{k+1}\right\|^{2}+\left\|\theta_{v}^{k+1}\right\|^{2}+\kappa\left|\theta_{u}^{k+1}\right|_{\frac{\alpha}{2}}^{2} \leq C N^{-2 \alpha}
$$

which implies that we have completed the mathematics induction. Therefore $(5.6)$ holds and then (5.7) is obtained similar as (5.20). We have completed the proof.

\section{The proof of the Theorem 3.3}

For convenience, we denote

$$
\begin{aligned}
& e_{N u}^{n}=u^{n}-u_{N}^{n}=u^{n}-P_{N} u^{n}+P_{N} u^{n}-u_{N}^{n}=\eta_{u}^{n}+\xi_{u}^{n}, \\
& e_{N v}^{n}=v^{n}-v_{N}^{n}=v^{n}-P_{N} v^{n}+P_{N} v^{n}-v_{N}^{n}=\eta_{v}^{n}+\xi_{v}^{n} .
\end{aligned}
$$

In the convergence analysis, we use the mathematical induction method. From (1.1)-(1.2), (4.17)(4.19) and 3.13 - 3.15), the error equations at $t=t_{\frac{1}{2}}$ are the following:

$$
\left(\frac{\tilde{\xi}_{u}^{\frac{1}{2}}}{\tau / 2}, \psi\right)=\left(\tilde{\xi}_{v}^{\frac{1}{2}}, \psi\right)+\left(\tilde{Q}_{1}^{\frac{1}{2}}, \psi\right)
$$




$$
\begin{aligned}
& \left(\frac{\tilde{\xi}_{v}^{\frac{1}{2}}}{\tau / 2}, \varphi\right)+\kappa\left((-\Delta)^{\frac{\alpha}{2}} \tilde{\xi}_{u}^{\frac{1}{2}}, \varphi\right)+\gamma_{1}\left((-\Delta)^{\frac{\alpha}{2}} \tilde{\xi}_{v}^{\frac{1}{2}}, \varphi\right)+\gamma_{2}\left(\tilde{\xi}_{v}^{\frac{1}{2}}, \varphi\right)+\left(\tilde{R}_{2}^{\frac{1}{2}}, \varphi\right)=\left(\tilde{Q}_{2}^{\frac{1}{2}}, \varphi\right), \\
& \frac{\tilde{e}_{r}^{\frac{1}{2}}}{\tau / 2}=\tilde{F}_{1}^{\frac{1}{2}}+\tilde{Q}_{3}^{\frac{1}{2}}
\end{aligned}
$$

where

$$
\begin{aligned}
& \tilde{R}_{2}^{\frac{1}{2}}=\tilde{r}^{\frac{1}{2}} \frac{F^{\prime}\left(u^{0}\right)}{\sqrt{E\left(u^{0}\right)}}-\tilde{R}^{\frac{1}{2}} \frac{F^{\prime}\left(u_{N}^{0}\right)}{\sqrt{E\left(u_{N}^{0}\right)}}=\tilde{e}_{r}^{\frac{1}{2}} \frac{F^{\prime}\left(u^{0}\right)}{\sqrt{E\left(u^{0}\right)}}+\tilde{R}^{\frac{1}{2}}\left(\frac{F^{\prime}\left(u^{0}\right)}{\sqrt{E\left(u^{0}\right)}}-\frac{F^{\prime}\left(u_{N}^{0}\right)}{\sqrt{E\left(u_{N}^{0}\right)}}\right), \\
& \tilde{F}_{1}^{\frac{1}{2}}=\frac{1}{2 \sqrt{E\left(u^{0}\right)}}\left(F^{\prime}\left(u^{0}\right), \frac{\tilde{u}^{\frac{1}{2}}-u^{0}}{\tau / 2}\right)-\frac{1}{2 \sqrt{E\left(u_{N}^{0}\right)}}\left(F^{\prime}\left(u_{N}^{0}\right), \frac{\tilde{u}_{N}^{\frac{1}{2}}-u_{N}^{0}}{\tau / 2}\right) \\
& =\left(\frac{1}{2 \sqrt{E\left(u^{0}\right)}} F^{\prime}\left(u^{0}\right)-\frac{1}{2 \sqrt{E\left(u_{N}^{0}\right)}} F^{\prime}\left(u_{N}^{0}\right), \frac{\tilde{u}^{\frac{1}{2}}-u^{0}}{\tau / 2}\right)+\frac{1}{2 \sqrt{E\left(u_{N}^{0}\right)}}\left(F^{\prime}\left(u_{N}^{0}\right), \frac{\tilde{e}_{u}^{\frac{1}{2}}-e_{u}^{0}}{\tau / 2}\right) .
\end{aligned}
$$

Letting $\psi=2 \tau \tilde{\xi}_{u}^{\frac{1}{2}}$ and $\varphi=2 \tau \tilde{\xi}_{v}^{\frac{1}{2}}$ in $6.17-(6.2)$, and multiplying $2 \tau \tilde{e}_{r}^{\frac{1}{2}}$ on the both sides of (6.3), and using

$$
\left(\tilde{\xi}_{u}^{\frac{1}{2}},(-\Delta)^{\frac{\alpha}{2}} \tilde{\xi}_{u}^{\frac{1}{2}}\right)=2 \tau\left(\tilde{\xi}_{v}^{\frac{1}{2}},(-\Delta)^{\frac{\alpha}{2}} \tilde{\xi}_{u}^{\frac{1}{2}}\right)+2 \tau\left(\tilde{Q}_{1}^{\frac{1}{2}},(-\Delta)^{\frac{\alpha}{2}} \tilde{\xi}_{u}^{\frac{1}{2}}\right)
$$

we have

$$
\begin{aligned}
& \left\|\tilde{\xi}_{u}^{\frac{1}{2}}\right\|^{2}=2 \tau\left(\tilde{\xi}_{\xi}^{\frac{1}{2}}, \tilde{\xi}_{u}^{\frac{1}{2}}\right)+2 \tau\left(\tilde{Q}_{1}^{\frac{1}{2}}, \tilde{\xi}_{u}^{\frac{1}{2}}\right), \\
& \left\|\tilde{\xi}_{v}^{\frac{1}{2}}\right\|^{2}+\kappa\left(\left.\tilde{\xi}_{u}^{\frac{1}{2}}\right|_{\frac{\alpha}{2}} ^{2}+\left.2 \tau \gamma_{1} \tilde{\xi}^{\frac{1}{2}}\right|_{\frac{\alpha}{2}} ^{2}+2 \gamma_{2} \tau\left\|\tilde{\xi}_{v}^{\frac{1}{2}}\right\|^{2}\right. \\
& =2 \tau\left(\tilde{Q}_{2}^{\frac{1}{2}}, \tilde{\xi}_{v}^{\frac{1}{2}}\right)-2 \tau\left(\tilde{R}_{2}^{\frac{1}{2}}, \tilde{\xi}_{v}^{\frac{1}{2}}\right)+2 \tau \kappa\left((-\Delta)^{\frac{\alpha}{4}} \tilde{Q}_{1}^{\frac{1}{2}},(-\Delta)^{\frac{\alpha}{4}} \tilde{\xi}_{u}^{\frac{1}{2}}\right), \\
& \left(\tilde{e}_{r}^{\frac{1}{2}}\right)^{2}=2 \tau\left(\tilde{F}_{1}^{\frac{1}{2}}, \tilde{e}_{r}^{\frac{1}{2}}\right)+2 \tau\left(\tilde{Q}_{3}^{\frac{1}{2}}, \tilde{e}_{r}^{\frac{1}{2}}\right) .
\end{aligned}
$$

From $6.47-6.8),\left|\tilde{\eta}_{u}^{\frac{1}{2}} / \frac{\tau}{2}\right| \leq N^{-m} \tau^{-1} / 2 \int_{t_{0}}^{t_{1}}\left\|u_{t}\right\|^{2} d s$, using the Cauchy-Schwarz inequality and Young's inequality, we could have

$$
\left|\tilde{e}_{r}^{\frac{1}{2}}\right|^{2}+\left\|\tilde{\xi}_{u}^{\frac{1}{2}}\right\|^{2}+\left\|\tilde{\xi}_{v}^{\frac{1}{2}}\right\|^{2}+\kappa\left|\tilde{\xi}_{u}^{\frac{1}{2}}\right|_{\frac{\alpha}{2}}^{2} \leq C \tau K_{*},
$$

where $K_{*}=\left\|\eta_{u}^{0}\right\|^{2}+N^{-2 m} \tau^{-1} / 2 \int_{t_{0}}^{t_{1}}\left\|u_{t}\right\|^{2} d s+\left\|\tilde{Q}_{3}^{\frac{1}{2}}\right\|^{2}+\left\|\tilde{Q}_{1}^{\frac{1}{2}}\right\|^{2}+\left\|\tilde{Q}_{2}^{\frac{1}{2}}\right\|^{2}+\left|\tilde{Q}_{1}^{\frac{1}{2}}\right|_{\alpha / 2}^{2}+\tau^{3} \int_{t_{0}}^{t_{1}}\left\|\frac{\partial^{3} u}{\partial t^{3}}(s)\right\|^{2} d s$. Then we get $\left|\tilde{e}_{r}^{\frac{1}{2}}\right|^{2}+\left\|\tilde{\xi}_{u}^{\frac{1}{2}}\right\|^{2}+\left\|\tilde{\xi}_{v}^{\frac{1}{2}}\right\|^{2}+\kappa\left|\tilde{\xi}_{u}^{\frac{1}{2}}\right|_{\frac{\alpha}{2}}^{2} \leq C\left(\tau^{3}+N^{-2 m}\right)$.

Then, we prove the equation (3.20) holds for $n=1$. From (1.1)-(1.2), (4.14)-(4.16) and (3.9)(3.11), the error equations for $n=0$ follow that

$$
\begin{aligned}
& \left(\delta_{t} \xi_{u}^{\frac{1}{2}}, \psi\right)=\left(\bar{\xi}_{v}^{\frac{1}{2}}, \psi\right)+\left(Q_{1}^{\frac{1}{2}}, \psi\right), \\
& \left(\delta_{t} \xi_{v}^{\frac{1}{2}}, \varphi\right)+\kappa\left((-\Delta)^{\frac{\alpha}{2}} \bar{\xi}_{u}^{\frac{1}{2}}, \varphi\right)+\gamma_{1}\left((-\Delta)^{\frac{\alpha}{2}} \bar{\xi}_{v}^{\frac{1}{2}}, \varphi\right)+\gamma_{2}\left(\bar{\xi}_{v}^{\frac{1}{2}}, \varphi\right)+\left(R_{2}^{\frac{1}{2}}, \varphi\right)=\left(Q_{2}^{\frac{1}{2}}, \varphi\right),
\end{aligned}
$$




$$
\delta_{t} e_{r}^{\frac{1}{2}}=\bar{F}^{\frac{1}{2}}+Q_{3}^{\frac{1}{2}}
$$

where

$$
\begin{aligned}
& R_{2}^{\frac{1}{2}}=\bar{r}^{\frac{1}{2}} \frac{F^{\prime}\left(\tilde{u}^{\frac{1}{2}}\right)}{\sqrt{E\left(\tilde{u}^{\frac{1}{2}}\right)}}-\bar{R}^{\frac{1}{2}} \frac{F^{\prime}\left(\tilde{u}_{N}^{n+\frac{1}{2}}\right)}{\sqrt{E\left(\tilde{u}_{N}^{\frac{1}{2}}\right)}}, \\
& \bar{F}^{\frac{1}{2}}=\frac{1}{2 \sqrt{E\left(\tilde{u}^{n+\frac{1}{2}}\right)}}\left(F^{\prime}\left(\tilde{u}^{\frac{1}{2}}\right), \delta_{t} u^{\frac{1}{2}}\right)-\frac{1}{2 \sqrt{E\left(\tilde{u}_{N}^{\frac{1}{2}}\right)}}\left(F^{\prime}\left(\tilde{u}_{N}^{\frac{1}{2}}\right), \delta_{t} u_{N}^{\frac{1}{2}}\right) .
\end{aligned}
$$

Since the proof for $n=0$ is similar to the following proof, we just presents the results for $\mathrm{k}=0$. It follows that

$$
\begin{aligned}
& \left|e_{r}^{1}\right|^{2}+\left\|\xi_{u}^{1}\right\|^{2}+\left\|\xi_{v}^{1}\right\|^{2}+\kappa\left|\xi_{u}^{1}\right|_{\frac{\alpha}{2}}^{2} \leq C \tau\left(\left\|\eta_{v}^{0}\right\|^{2}+\left\|\eta_{u}^{0}\right\|^{2}+\left\|Q_{2}^{\frac{1}{2}}\right\|^{2}+\left\|Q_{1}^{\frac{1}{2}}\right\|^{2}+\left\|Q_{3}^{\frac{1}{2}}\right\|^{2}+\left|Q_{1}^{\frac{1}{2}}\right|_{\alpha / 2}^{2}+\left\|\tilde{\eta}_{u}^{\frac{1}{2}}\right\|^{2}+\left\|\tilde{\xi}_{u}^{\frac{1}{2}}\right\|^{2}\right. \\
& \left.+\tau^{3} \int_{t_{0}}^{t_{1}}\left\|\frac{\partial^{3} u}{\partial t^{3}}(s)\right\|^{2} d s+C N^{-2 m} \tau^{-1} \int_{t_{0}}^{t_{1}}\left\|u_{t}\right\|^{2} d s\right) \leq C\left(\tau^{4}+N^{-2 m}\right) .
\end{aligned}
$$

Next, we assume the 3.20 holds for $n=2, \ldots, k$ and will prove that the equation 3.20$)$ holds for $n=k+1$. From (1.1)-(1.2), (4.14)-(4.16) and (3.9)-(3.11), we can get

$$
\begin{aligned}
& \left(\delta_{t} \xi_{u}^{n+\frac{1}{2}}, \psi\right)=\left(\bar{\xi}_{v}^{n+\frac{1}{2}}, \psi\right)+\left(Q_{1}^{n+\frac{1}{2}}, \psi\right), \\
& \left(\delta_{t} \xi_{v}^{n+\frac{1}{2}}, \varphi\right)+\kappa\left((-\Delta)^{\frac{\alpha}{2}} \bar{\xi}_{u}^{n+\frac{1}{2}}, \varphi\right)+\gamma_{1}\left((-\Delta)^{\frac{\alpha}{2}} \bar{\xi}_{v}^{n+\frac{1}{2}}, \varphi\right)+\gamma_{2}\left(\bar{\xi}_{v}^{n+\frac{1}{2}}, \varphi\right)+\left(R_{2}^{n+\frac{1}{2}}, \varphi\right)=\left(Q_{2}^{n+\frac{1}{2}}, \varphi\right), \\
& \delta_{t} e_{r}^{n+\frac{1}{2}}=\bar{F}^{n+\frac{1}{2}}+Q_{3}^{n+\frac{1}{2}}
\end{aligned}
$$

where

$$
\begin{aligned}
& R_{2}^{n+\frac{1}{2}}=\bar{r}^{n+\frac{1}{2}} \frac{F^{\prime}\left(\tilde{u}^{n+\frac{1}{2}}\right)}{\sqrt{E\left(\tilde{u}^{n+\frac{1}{2}}\right)}}-\bar{R}^{n+\frac{1}{2}} \frac{F^{\prime}\left(\tilde{u}_{N}^{n+\frac{1}{2}}\right)}{\sqrt{E\left(\tilde{u}_{N}^{n+\frac{1}{2}}\right)}}, \\
& \bar{F}^{n+\frac{1}{2}}=\frac{1}{2 \sqrt{E\left(\tilde{u}^{n+\frac{1}{2}}\right)}}\left(F^{\prime}\left(\tilde{u}^{n+\frac{1}{2}}\right), \delta_{t} u^{n+\frac{1}{2}}\right)-\frac{1}{2 \sqrt{E\left(\tilde{u}_{N}^{n+\frac{1}{2}}\right)}}\left(F^{\prime}\left(\tilde{u}_{N}^{n+\frac{1}{2}}\right), \delta_{t} u_{N}^{n+\frac{1}{2}}\right) .
\end{aligned}
$$

Letting $\psi=2 \tau \bar{\xi}_{u}^{n+\frac{1}{2}}$ and $\varphi=2 \tau \bar{\xi}_{v}^{n+\frac{1}{2}}$ in 6.14 -6.15 , and multiplying $2 \tau \bar{e}_{r}^{n+\frac{1}{2}}$ on the both sides of 6.16, and using

$$
\left(\delta_{t} \xi_{u}^{n+\frac{1}{2}},(-\Delta)^{\frac{\alpha}{2}} \bar{\xi}_{u}^{n+\frac{1}{2}}\right)=\left(\xi_{v}^{n+\frac{1}{2}},(-\Delta)^{\frac{\alpha}{2}} \bar{\xi}_{u}^{n+\frac{1}{2}}\right)+\left(Q_{1}^{n+\frac{1}{2}},(-\Delta)^{\frac{\alpha}{2}} \bar{\xi}_{u}^{n+\frac{1}{2}}\right)
$$

we can get

$$
\begin{aligned}
& \left\|\xi_{u}^{n+1}\right\|^{2}-\left\|\xi_{u}^{n}\right\|^{2}=2 \tau\left(\bar{\xi}_{v}^{n+\frac{1}{2}}, \bar{\xi}_{u}^{n+\frac{1}{2}}\right)+2 \tau\left(Q_{1}^{n+\frac{1}{2}}, \bar{\xi}_{u}^{n+\frac{1}{2}}\right), \\
& \left\|\xi_{v}^{n+1}\right\|^{2}-\left\|\xi_{v}^{n}\right\|^{2}+\kappa\left(\left|\xi_{u}^{n+1}\right|_{\frac{\alpha}{2}}^{2}-\left|\xi_{u}^{n}\right|_{\frac{\alpha}{2}}^{2}\right)+2 \tau \gamma_{1}\left|\bar{\xi}_{v}^{n+\frac{1}{2}}\right|_{\frac{\alpha}{2}}^{2}+2 \gamma_{2} \tau\left\|\bar{\xi}_{v}^{n+\frac{1}{2}}\right\|^{2} \\
& =2 \tau\left(Q_{2}^{n+\frac{1}{2}}, \bar{\xi}_{v}^{n+\frac{1}{2}}\right)-2 \tau\left(R_{2}^{n+\frac{1}{2}}, \bar{\xi}_{v}^{n+\frac{1}{2}}\right)+2 \tau \kappa\left((-\Delta)^{\frac{\alpha}{4}} Q_{1}^{n+\frac{1}{2}},(-\Delta)^{\frac{\alpha}{4}} \bar{\xi}_{u}^{n+\frac{1}{2}}\right),
\end{aligned}
$$




$$
\left(e_{r}^{n+1}\right)^{2}-\left(e_{r}^{n}\right)^{2}=2 \tau\left(\bar{F}^{n+\frac{1}{2}}, \bar{e}_{r}^{n+\frac{1}{2}}\right)+2 \tau\left(Q_{3}^{n+\frac{1}{2}}, \bar{e}_{r}^{n+\frac{1}{2}}\right)
$$

where

$$
\begin{aligned}
& R_{2}^{n+\frac{1}{2}}=r^{n+\frac{1}{2}}\left(\frac{F^{\prime}\left(\tilde{u}^{n+\frac{1}{2}}\right)}{\sqrt{E\left(\tilde{u}^{n+\frac{1}{2}}\right)}}-\frac{F^{\prime}\left(\tilde{u}_{N}^{n+\frac{1}{2}}\right)}{\sqrt{E\left(\tilde{u}_{N}^{n+\frac{1}{2}}\right)}}\right)+e_{r}^{n+\frac{1}{2}} \frac{F^{\prime}\left(\tilde{u}_{N}^{n+\frac{1}{2}}\right)}{\sqrt{E\left(\tilde{u}_{N}^{n+\frac{1}{2}}\right)}}, \\
& \bar{F}^{n+\frac{1}{2}}=\left(\frac{1}{2 \sqrt{E\left(\tilde{u}^{n+\frac{1}{2}}\right)}} F^{\prime}\left(\tilde{u}^{n+\frac{1}{2}}\right)-\frac{1}{\left.2 \sqrt{E\left(\tilde{u}_{N}^{n+\frac{1}{2}}\right.}\right)} F^{\prime}\left(\tilde{u}_{N}^{n+\frac{1}{2}}\right), \delta_{t} u^{n+\frac{1}{2}}\right)+\left(\frac{1}{2 \sqrt{E\left(\tilde{u}_{N}^{n+\frac{1}{2}}\right)}} F^{\prime}\left(\tilde{u}_{N}^{n+\frac{1}{2}}\right), \delta_{t} \bar{e}_{u}^{n+\frac{1}{2}}\right), \\
& \frac{1}{\sqrt{E\left(\tilde{u}^{n+\frac{1}{2}}\right)}} F^{\prime}\left(\tilde{u}^{n+\frac{1}{2}}\right)-\frac{1}{\left.\sqrt{E\left(\tilde{u}_{N}^{n+\frac{1}{2}}\right.}\right)} F^{\prime}\left(\tilde{u}_{N}^{n+\frac{1}{2}}\right)= \\
& \frac{F^{\prime}\left(\tilde{u}^{n+\frac{1}{2}}\right)-F^{\prime}\left(\tilde{u}_{N}^{n+\frac{1}{2}}\right)}{\sqrt{E\left(\tilde{u}^{n+\frac{1}{2}}\right)}}+\frac{F^{\prime}\left(\tilde{u}_{N}^{n+\frac{1}{2}}\right)\left(E\left(\tilde{u}^{n+\frac{1}{2}}\right)-E\left(\tilde{u}_{N}^{n+\frac{1}{2}}\right)\right)}{\sqrt{E\left(\tilde{u}_{N}^{n+\frac{1}{2}}\right)} \sqrt{E\left(\tilde{u}^{n+\frac{1}{2}}\right)}\left(\sqrt{E\left(\tilde{u}^{n+\frac{1}{2}}\right)}+\sqrt{E\left(\tilde{u}_{N}^{n+\frac{1}{2}}\right)},\right.} \\
& \left(\frac{1}{2 \sqrt{E\left(\tilde{u}_{N}^{n+\frac{1}{2}}\right)}} F^{\prime}\left(\tilde{u}_{N}^{n+\frac{1}{2}}\right), \delta_{t} \bar{e}_{u}^{n+\frac{1}{2}}\right)=\left(\frac{1}{\left.2 \sqrt{E\left(\tilde{u}_{N}^{n+\frac{1}{2}}\right.}\right)} F^{\prime}\left(\tilde{u}_{N}^{n+\frac{1}{2}}\right), \delta_{t} \bar{\eta}_{u}^{n+\frac{1}{2}}+\delta_{t} \bar{\xi}_{u}^{n+\frac{1}{2}}\right) \\
& =\left(\frac{1}{2 \sqrt{E\left(\tilde{u}_{N}^{n+\frac{1}{2}}\right.}} F^{\prime}\left(\tilde{u}_{N}^{n+\frac{1}{2}}\right), \delta_{t} \bar{\eta}_{u}^{n+\frac{1}{2}}\right)+\left(\frac{1}{2 \sqrt{E\left(\tilde{u}_{N}^{n+\frac{1}{2}}\right.}} F^{\prime}\left(\tilde{u}_{N}^{n+\frac{1}{2}}\right), \bar{\xi}_{v}^{n+\frac{1}{2}}+Q_{1}^{n+\frac{1}{2}}\right) .
\end{aligned}
$$

From Theorem 5.1, we get $u_{N}^{n}$ and $\tilde{u}_{N}^{n+\frac{1}{2}}$ are bounded. Following the proof of 4.70) and from (6.23)-6.25), 4.68), we obtain

$$
\begin{gathered}
\left|\left(\frac{1}{2 \sqrt{E\left(\tilde{u}_{N}^{n+\frac{1}{2}}\right)}} F^{\prime}\left(\tilde{u}_{N}^{n+\frac{1}{2}}\right), \delta_{t} \bar{\eta}_{u}^{n+\frac{1}{2}}\right)\right| \leq C\left(\left\|\tilde{u}_{N}^{n+\frac{1}{2}}\right\|_{\infty}\right)\left|\delta_{t} \eta_{u}^{n+\frac{1}{2}}\right| \leq C N^{-m} \tau^{-1} \int_{t_{n}}^{t_{n+1}}\left\|u_{t}^{n+1 / 2}\right\| d s, \\
\tau\left|\left(R_{2}^{n+\frac{1}{2}}, \bar{\xi}_{v}^{n+\frac{1}{2}}\right)\right| \leq C \tau\left(\left\|\xi_{v}^{n+1}\right\|^{2}+\left\|\xi_{v}^{n}\right\|^{2}+\left(e_{r}^{n+1}\right)^{2}+\left(e_{r}^{n}\right)^{2}+\left\|\tilde{\xi}_{u}^{n+\frac{1}{2}}\right\|^{2}\right)+ \\
C \tau\left(\left\|\eta_{v}^{n+1}\right\|^{2}+\left\|\eta_{v}^{n}\right\|^{2}+\left\|\eta_{u}^{n}\right\|^{2}+\left\|\eta_{u}^{n-1}\right\|^{2}\right), \\
\tau\left|\left(\bar{F}^{n+\frac{1}{2}}, \bar{e}_{r}^{n+\frac{1}{2}}\right)\right| \leq C \tau\left(\left\|\tilde{\xi}_{u}^{n+\frac{1}{2}}\right\|^{2}+\left\|\tilde{\eta}_{u}^{n+\frac{1}{2}}\right\|^{2}+\left\|\xi_{v}^{n+1}\right\|^{2}+\left\|\xi_{v}^{n}\right\|^{2}+\left|e_{r}^{n+1}\right|^{2}+\left|e_{r}^{n}\right|^{2}\right. \\
\left.+\left\|\eta_{v}^{n+1}\right\|^{2}+\left\|\eta_{v}^{n}\right\|^{2}+\left\|Q_{1}^{n+\frac{1}{2}}\right\|^{2}\right)+C \tau^{4} \int_{t_{n}}^{t_{n+1}}\left\|\frac{\partial^{3} u}{\partial t^{3}}(s)\right\|^{2} d s+C N^{-2 m} \int_{t_{n}}^{t_{n+1}}\left\|u_{t}^{n+1 / 2}\right\|^{2} d s .
\end{gathered}
$$

For convenience, we let $E^{n}:=\left|e_{r}^{n}\right|^{2}+\left\|\xi_{u}^{n}\right\|^{2}+\left\|\xi_{v}^{n}\right\|^{2}+\kappa\left|\xi_{u}^{n}\right|_{\frac{\alpha}{2}}^{2}$. Then, from 6.19)-6.28, we get

$$
E^{n+1}-E^{n} \leq C \tau\left(E^{n+1}+E^{n}\right)+C \tau \Re,
$$

where $\Re=\left\|\eta_{v}^{n+1}\right\|^{2}+\left\|\eta_{v}^{n}\right\|^{2}+\left\|\eta_{u}^{n}\right\|^{2}+\left\|\eta_{u}^{n-1}\right\|^{2}+\left\|Q_{2}^{n+\frac{1}{2}}\right\|^{2}+\left\|Q_{1}^{n+\frac{1}{2}}\right\|^{2}+\left\|Q_{3}^{n+\frac{1}{2}}\right\|^{2}+\left|Q_{1}^{n+\frac{1}{2}}\right|_{\alpha / 2}^{2}+\left\|\tilde{\eta}_{u}^{n+\frac{1}{2}}\right\|^{2}+$ $\left\|\tilde{\xi}_{u}^{n+\frac{1}{2}}\right\|^{2}+\tau^{3} \int_{t_{n}}^{t_{n+1}}\left\|\frac{\partial^{3} u}{\partial t^{3}}(s)\right\|^{2} d s+C N^{-2 m} \tau^{-1} \int_{t_{n}}^{t_{n+1}}\left\|u_{t}^{n+1 / 2}\right\|^{2} d s$. By virtue of discrete Gronwall's inequality, one arrives at $E^{k+1} \leq C\left(N^{-2 m}+\tau^{4}\right)$, which shows that the equation 3.20 ) holds for $n=k+1$ 
and we have completed the mathematics induction. Thus, without losing generality, we conclude

$$
\left|e_{r}^{n}\right|^{2}+\left\|\xi_{u}^{n}\right\|^{2}+\left\|\xi_{v}^{n}\right\|^{2}+\kappa\left|\xi_{u}^{n}\right|_{\frac{\alpha}{2}}^{2} \leq C\left(\tau^{4}+N^{-2 m}\right) \text {. }
$$

Using Lemma 2.5 and the triangle inequality, we have

$$
\left\|u^{n}-u_{N}^{n}\right\|^{2}+\left\|v^{n}-v_{N}^{n}\right\|^{2}+\left|r^{n}-R^{n}\right|^{2} \leq C\left(\tau^{4}+N^{-2 m}\right), \kappa\left|u^{n}-u_{N}^{n}\right|_{\frac{\alpha}{2}}^{2} \leq C\left(\tau^{4}+N^{\alpha-2 m}\right) .
$$

The proof of Theorem 3.3 is end when $\tau \leq \tau^{*}, N \geq N^{*}$.

For the other three cases, we denote $S_{0}:=\left(\tau^{*}\right)^{4}+\left(N^{*}\right)^{-2 m}$.

1. When $\tau \geq \tau^{*}, N \leq N^{*}$, there holds $\tau^{4}+N^{-2 m} \geq S_{0}$. By using the continuous and discrete energy dissipation, i.e., $H(t) \leq H\left(t_{0}\right)$ and $\mathbf{H}^{n} \leq \mathbf{H}^{0}$, we can get

$$
\begin{aligned}
& \left\|u^{n}-u_{N}^{n}\right\|^{2}+\left\|v^{n}-v_{N}^{n}\right\|^{2}+\left|r^{n}-R^{n}\right|^{2} \\
& \leq\left\|u^{n}\right\|^{2}+\left\|u_{N}^{n}\right\|^{2}+\left\|v^{n}\right\|^{2}+\left\|v_{N}^{n}\right\|^{2}+\left|r^{n}\right|^{2}+\left|R^{n}\right|^{2}+\kappa\left|u^{n}\right|_{\frac{\alpha}{2}}^{2}+\kappa\left|u_{N}^{n}\right|_{\frac{\alpha}{2}}^{2} \\
& \leq\left(\frac{2}{\kappa}+2\right)\left(H\left(t_{0}\right)+\mathbf{H}^{0}\right) \leq C\left(\tau^{4}+N^{-2 m}\right), \\
& \kappa\left|u^{n}-u_{N}^{n}\right|_{\frac{\alpha}{2}}^{2} \leq \kappa\left|u^{n}\right|_{\frac{\alpha}{2}}^{2}+\kappa\left|u_{N}^{n}\right|_{\frac{\alpha}{2}}^{2} \leq\left(\frac{2}{\kappa}+2\right)\left(H\left(t_{0}\right)+\mathbf{H}^{0}\right) \leq C\left(\tau^{4}+N^{\alpha-2 m}\right),
\end{aligned}
$$

where $C=\left(\frac{2}{\kappa}+2\right)\left(H\left(t_{0}\right)+\mathbf{H}^{0}\right) / S_{0}$.

2. When $\tau \leq \tau^{*}, N \leq N^{*}$, it obviously follows that

$$
C_{1} N^{-2 m} \geq S_{0} \geq \tau^{4}+\left(N^{*}\right)^{-2 m},
$$

where $C_{1}=\frac{\left(N^{*}\right)^{-2 m}+\left(\tau^{*}\right)^{4}}{\left(N^{*}\right)^{-2 m}}$. In this case, we also have

$$
\begin{aligned}
& \left\|u^{n}-u_{N}^{n}\right\|^{2}+\left\|v^{n}-v_{N}^{n}\right\|^{2}+\left|r^{n}-R^{n}\right|^{2} \leq\left(\frac{2}{\kappa}+2\right)\left(H\left(t_{0}\right)+\mathbf{H}^{0}\right) \leq C\left(\tau^{4}+N^{-2 m}\right), \\
& \kappa\left|u^{n}-u_{N}^{n}\right|_{\frac{\alpha}{2}} \leq C\left(\tau^{4}+N^{\alpha-2 m}\right),
\end{aligned}
$$

where $C=\left(\frac{2}{\kappa}+2\right)\left(H\left(t_{0}\right)+\mathbf{H}^{0}\right) C_{1} / S_{0}$.

3. When $\tau \geq \tau^{*}, N \geq N^{*}$, there holds

$$
C_{2} \tau^{4} \geq S_{0} \geq\left(\tau^{*}\right)^{4}+(N)^{-2 m},
$$

where $C_{2}=\frac{\left(N^{*}\right)^{-2 m}+\left(\tau^{*}\right)^{4}}{\left(\tau^{*}\right)^{4}}$. In this case, we also have

$$
\begin{aligned}
& \left\|u^{n}-u_{N}^{n}\right\|^{2}+\left\|v^{n}-v_{N}^{n}\right\|^{2}+\left|r^{n}-R^{n}\right|^{2} \leq\left(\frac{2}{\kappa}+2\right)\left(H\left(t_{0}\right)+\mathbf{H}^{0}\right) \leq C\left(\tau^{4}+N^{-2 m}\right), \\
& \kappa\left|u^{n}-u_{N}^{n}\right|_{\frac{\alpha}{2}} \leq C\left(\tau^{4}+N^{\alpha-2 m}\right),
\end{aligned}
$$

where $C=\left(\frac{2}{\kappa}+2\right)\left(H\left(t_{0}\right)+\mathbf{H}^{0}\right) C_{2} / S_{0}$.

In this work, $\kappa$ is suitable positive constant and does not effect the error estimate of our scheme. Therefore, we complete the proof of Theorem 3.3 .

Remark 6.1. Since the FGWE reduces to classical generalized wave equation when $\alpha=2$, the unconditional energy dissipation and convergence analysis for the FGWE in this paper can naturally be applied to that of conventional generalized wave equation. 


\section{Numerical experiments}

In this section, we will present some numerical examples to confirm the discrete energy dissipation property and the accuracy of the full discrete SAV Fourier spectral schemes.

Example 1. In this example, we take $\Omega=(-16,16) \times(-16,16), T=1, \kappa=1$. we consider $F(u)=$ $1-\cos u$, and take the initial value $u(x, y, 0)=\sin (\pi x / 16) \cos (\pi y / 16)$ and the corresponding $u_{t}(x, y, 0)=0$.

- case 1: $\gamma_{1}=0, \gamma_{2}=0$.

- case 2: $\gamma_{1}=1, \gamma_{2}=1$.

Since we have not the exact solution, and thus we choose sufficiently small time step $K=1000$ and $N=256$ to get 'exact' solution. For simplicity, Table 1 for case 1 just presents the errors of $\left\|e_{u}^{n}\right\|_{\infty},\left\|e_{v}^{n}\right\|_{\infty},\left\|e_{r}^{n}\right\|_{\infty}$ in time and show that the fully discrete SAV scheme is second order accuracy in time. Table 2 presents the spatial error of case 1 and we get spectral accuracy. Similar to the computational accuracy for case 1, we also present the temporal accuracy in Table 3 and spatial accuracy for case 2 in Table 4 . Next, we will verify the conservation or dissipation property of the SAV Fourier spectral method. Fig. 1 shows the time evolution of the discrete energy $\mathbf{H}^{n}$ with different values $\gamma_{1}$ and $\gamma_{2}$ for $\alpha=1.2$ and $\alpha=1.8$ and associate errors of $\mathbf{H}^{n}$ for the conservation case is presented in Fig. 2. We observe that the damping parameters $\gamma_{1}$ and $\gamma_{2}$ efficiently effect the dissipation property for SAV scheme for long time simulation.

Example 2.

$$
\begin{aligned}
& u_{t t}+(-\Delta)^{\frac{\alpha}{2}} u+\gamma_{1}(-\Delta)^{\frac{\alpha}{2}} u_{t}+\gamma_{2} u_{t}+F^{\prime}(u)=0,(x, y, t) \in \Omega \times(0, T], \\
& u(x, y, 0)=\frac{1}{2} \arctan \left(\exp \left(-\sqrt{x^{2}+y^{2}}\right)\right), u_{t}(x, y, 0)=0,
\end{aligned}
$$

where $\Omega=(-10,10) \times(-10,10)$. We present the profiles of numerical solution $u_{N}$ with different potential energy $F(u)=u^{2}\left(\frac{1}{4} u^{2}-\frac{1}{2}\right)$ for $\alpha=1.2$ and different values of $\gamma_{1}=\gamma_{2}$ at $T=8$, which shows that $\gamma_{1}, \gamma_{2}$ have impacts on the profiles of wave. In addition, we need to confirm the fully discrete energy dissipation-preserving or conservation property of numerical solution. Figs. 5 presents the discrete energy $\mathbf{H}^{n}$ with different values of $\gamma_{1}$ and $\gamma_{2}$ in long time simulation. They also show that the influence of coefficients $\gamma_{1}$ and $\gamma_{2}$ of the damping term, i.e., when $\gamma_{1}, \gamma_{2} \rightarrow 0$, the energy discrete energy $\mathbf{H}^{n}$ decays more slowly.

\section{Conclusion}

In this paper, we proposed a linearly implicit scheme to solve the nonlinear fractional general wave equation, and we used the SAV approach in time and Fourier spectral method in space. The resulting system is a linear system at each time step and FFT solver can be adopted in space, so that the scheme could be efficient to implement. The energy conservation or dissipation property of the scheme was strictly proved. In addition, we rigorously proved the unconditional convergence for the scheme by the temporal-spatial error splitting technique and got the second order accuracy in time and spectral accuracy in space. Finally, some numerical results were given to confirm our theoretical analysis. 
Table 1: The $L^{\infty}$ errors of $u, v$ and $r$ in time for case 1 of Example 1 at time $T=1$.

\begin{tabular}{ccccccc}
\hline \multicolumn{5}{c}{$\alpha=1.2, N=256$} \\
\hline$\Delta t$ & $\left\|e_{u}\right\|_{\infty}$ & rate & $\left\|e_{v}\right\|_{\infty}$ & rate & $\left\|e_{r}\right\|_{\infty}$ & rate \\
\hline $1 / 10$ & $5.4953 \mathrm{e}-05$ & & $3.8436 \mathrm{e}-05$ & & $7.0286 \mathrm{e}-05$ & \\
$1 / 20$ & $1.3671 \mathrm{e}-05$ & 2.0228 & $9.4748 \mathrm{e}-06$ & 2.0262 & $1.8739 \mathrm{e}-05$ & 1.9071 \\
$1 / 40$ & $3.4055 \mathrm{e}-06$ & 2.0400 & $2.4215 \mathrm{e}-06$ & 2.0200 & $4.8090 \mathrm{e}-06$ & 1.9622 \\
\hline \multicolumn{5}{c}{$\alpha=1.5, N=256$} \\
\hline$\Delta t$ & $\left\|e_{u}\right\|_{\infty}$ & rate & $\left\|e_{v}\right\|_{\infty}$ & rate & $\left\|e_{r}\right\|_{\infty}$ & rate \\
\hline $1 / 10$ & $2.6766 \mathrm{e}-05$ & \multicolumn{5}{c}{$4.0322 \mathrm{e}-05$} \\
$1 / 20$ & $6.5081 \mathrm{e}-06$ & 2.0400 & $1.0764 \mathrm{e}-05$ & 1.9053 & $2.5043 \mathrm{e}-05$ & 1.9186 \\
$1 / 40$ & $1.6014 \mathrm{e}-06$ & 2.0229 & $2.7703 \mathrm{e}-06$ & 1.9581 & $6.4132 \mathrm{e}-06$ & 1.8011 \\
\hline \multicolumn{5}{c}{$\alpha=1.8, N=256$} \\
\hline$\Delta t$ & $\left\|e_{u}\right\|_{\infty}$ & rate & $\left\|e_{v}\right\|_{\infty}$ & rate & $\left\|e_{r}\right\|_{\infty}$ & rate \\
\hline $1 / 10$ & $1.1679 \mathrm{e}-05$ & & $4.4344 \mathrm{e}-05$ & & $1.0619 \mathrm{e}-04$ & \\
$1 / 20$ & $2.6560 \mathrm{e}-06$ & 2.0070 & $1.1815 \mathrm{e}-05$ & 2.0202 & $2.8057 \mathrm{e}-05$ & 1.9202 \\
$1 / 40$ & $6.2907 \mathrm{e}-07$ & 2.0051 & $3.0389 \mathrm{e}-06$ & 1.9681 & $7.1860 \mathrm{e}-06$ & 1.9650 \\
\hline \multicolumn{5}{c}{$\alpha=2.0, N=256$} \\
\hline$\Delta t$ & $\left\|e_{u}\right\|_{\infty}$ & rate & $\left\|e_{v}\right\|_{\infty}$ & rate & $\left\|e_{r}\right\|_{\infty}$ & rate \\
\hline $1 / 10$ & $6.0094 \mathrm{e}-06$ & & $4.6442 \mathrm{e}-05$ & & $1.1018 \mathrm{e}-04$ & \\
$1 / 20$ & $2.3176 \mathrm{e}-06$ & 1.3745 & $1.2362 \mathrm{e}-05$ & 1.9095 & $2.9115 \mathrm{e}-05$ & 1.9200 \\
$1 / 40$ & $6.8385 \mathrm{e}-07$ & 1.7608 & $3.1787 \mathrm{e}-06$ & 1.9594 & $7.4597 \mathrm{e}-06$ & 1.9645 \\
\hline \multicolumn{5}{c}{}
\end{tabular}

Table 2: The errors $\left|e_{u}\right| \frac{\alpha}{2}$ and $\left\|e_{u}\right\|$ in space for case 1 of Example 1 at time $T=1$.

\begin{tabular}{cccccc}
\hline & $\mathrm{N}$ & $\left\|e_{u}\right\|_{\frac{\alpha}{2}}$ & rate & $\left\|e_{v}\right\|$ & rate \\
\hline \multirow{5}{*}{$\alpha=1.2$} & 4 & $2.4856 \mathrm{e}-02$ & & $4.7569 \mathrm{e}-03$ & \\
& 8 & $1.2884 \mathrm{e}-03$ & 4.2699 & $1.6427 \mathrm{e}-04$ & 4.8558 \\
& 16 & $1.5766 \mathrm{e}-07$ & 12.9965 & $3.3921 \mathrm{e}-08$ & 12.2416 \\
& 32 & $3.6791 \mathrm{e}-10$ & 8.7432 & $3.6608 \mathrm{e}-11$ & 9.8558 \\
\hline \multirow{5}{*}{$\alpha=1.5$} & 4 & $2.0858 \mathrm{e}-02$ & & $4.9288 \mathrm{e}-03$ & \\
& 8 & $1.2541 \mathrm{e}-03$ & 4.0559 & $1.7487 \mathrm{e}-04$ & 4.8169 \\
& 16 & $1.7644 \mathrm{e}-07$ & 12.7952 & $3.6692 \mathrm{e}-08$ & 12.2185 \\
& 32 & $3.6622 \mathrm{e}-10$ & 8.9123 & $7.8163 \mathrm{e}-11$ & 8.8748 \\
\hline \multirow{4}{*}{$\alpha=1.8$} & 4 & $1.7418 \mathrm{e}-02$ & & $5.0534 \mathrm{e}-03$ & \\
& 8 & $1.2128 \mathrm{e}-03$ & 3.8442 & $1.8282 \mathrm{e}-04$ & 4.7888 \\
& 16 & $1.9317 \mathrm{e}-07$ & 12.6162 & $3.8413 \mathrm{e}-08$ & 12.2165 \\
& 32 & $3.3982 \mathrm{e}-10$ & 9.1509 & $7.3433 \mathrm{e}-11$ & 9.0310 \\
\hline \multirow{5}{*}{$\alpha=2.0$} & 4 & $1.5416 \mathrm{e}-02$ & & $5.1169 \mathrm{e}-03$ & \\
& 8 & $1.1831 \mathrm{e}-03$ & 3.7038 & $1.8693 \mathrm{e}-04$ & 4.7747 \\
& 16 & $2.0333 \mathrm{e}-07$ & 12.5065 & $3.9072 \mathrm{e}-08$ & 12.2241 \\
& 32 & $2.8541 \mathrm{e}-10$ & 9.4766 & $7.8163 \mathrm{e}-10$ & 5.6435 \\
\hline
\end{tabular}


Table 3: The $L^{\infty}$ errors of $u, v$ and $r$ in time for case 2 of Example 1 at time $T=1$.

\begin{tabular}{|c|c|c|c|c|c|c|}
\hline & \multicolumn{6}{|c|}{$\alpha=1.2, \quad N=256$} \\
\hline$\Delta t$ & $\left\|e_{u}\right\|_{\infty}$ & rate & $\left\|e_{v}\right\|_{\infty}$ & rate & $\left\|e_{r}\right\|_{\infty}$ & rate \\
\hline $1 / 10$ & $1.1203 \mathrm{e}-04$ & & $1.2852 \mathrm{e}-04$ & & $1.6015 \mathrm{e}-05$ & \\
\hline $1 / 20$ & $2.7924 \mathrm{e}-05$ & 2.0043 & $3.2131 \mathrm{e}-05$ & 1.9999 & $3.9645 \mathrm{e}-05$ & 2.0142 \\
\hline \multirow[t]{2}{*}{$1 / 40$} & $6.9647 \mathrm{e}-06$ & 2.0034 & $8.0258 \mathrm{e}-06$ & 2.0012 & $9.8517 \mathrm{e}-06$ & 2.0087 \\
\hline & \multicolumn{6}{|c|}{$\alpha=1.5, \quad N=256$} \\
\hline$\Delta t$ & $\left\|e_{u}\right\|_{\infty}$ & rate & $\left\|e_{v}\right\|_{\infty}$ & rate & $\left\|e_{r}\right\|_{\infty}$ & rate \\
\hline $1 / 10$ & $8.3862 \mathrm{e}-05$ & & $9.8031 \mathrm{e}-05$ & & $1.2404 \mathrm{e}-04$ & \\
\hline $1 / 20$ & $2.0836 \mathrm{e}-05$ & 2.0088 & $2.4533 e-05$ & 1.9985 & $3.0467 \mathrm{e}-05$ & 2.0255 \\
\hline \multirow[t]{2}{*}{$1 / 40$} & $5.1883 \mathrm{e}-06$ & 2.0057 & $6.1308 \mathrm{e}-06$ & 2.0005 & $7.5528 \mathrm{e}-06$ & 2.0121 \\
\hline & \multicolumn{6}{|c|}{$\alpha=1.8, \quad N=256$} \\
\hline$\Delta t$ & $\left\|e_{u}\right\|_{\infty}$ & rate & $\left\|e_{v}\right\|_{\infty}$ & rate & $\left\|e_{r}\right\|_{\infty}$ & rate \\
\hline $1 / 10$ & $6.4938 \mathrm{e}-05$ & & $7.9646 \mathrm{e}-05$ & & $1.0115 \mathrm{e}-04$ & \\
\hline $1 / 20$ & $1.6072 \mathrm{e}-05$ & 2.0144 & $1.9955 \mathrm{e}-05$ & 1.9985 & $2.4672 \mathrm{e}-05$ & 2.0355 \\
\hline \multirow[t]{2}{*}{$1 / 40$} & $3.9939 \mathrm{e}-06$ & 2.0087 & $6.1308 \mathrm{e}-06$ & 2.0005 & $6.0930 \mathrm{e}-06$ & 2.0177 \\
\hline & \multicolumn{6}{|c|}{$\alpha=2.0, \quad N=256$} \\
\hline$\Delta t$ & $\left\|e_{u}\right\|_{\infty}$ & rate & $\left\|e_{v}\right\|_{\infty}$ & rate & $\left\|e_{r}\right\|_{\infty}$ & rate \\
\hline $1 / 10$ & $5.5967 \mathrm{e}-05$ & & $7.1465 e-05$ & & $9.0572 \mathrm{e}-05$ & \\
\hline $1 / 20$ & $1.3813 \mathrm{e}-05$ & 2.0185 & $1.7920 \mathrm{e}-05$ & 1.9956 & $2.2004 \mathrm{e}-05$ & 2.0413 \\
\hline $1 / 40$ & $3.4273 \mathrm{e}-06$ & 2.0108 & $4.4825 \mathrm{e}-06$ & 1.9992 & $5.4268 \mathrm{e}-06$ & 2.0196 \\
\hline
\end{tabular}

Table 4: The errors $\left|e_{u}\right| \frac{\alpha}{2}$ and $\left\|e_{u}\right\|$ in space for case 2 of Example 1 at time $T=1$.

\begin{tabular}{cccccc}
\hline & $\mathrm{N}$ & $\left\|e_{u}\right\|_{\frac{\alpha}{2}}$ & rate & $\left\|e_{v}\right\|$ & rate \\
\hline \multirow{5}{*}{$\alpha=1.2$} & 4 & $1.6873 \mathrm{e}-02$ & & $2.6620 \mathrm{e}-03$ & \\
& 8 & $7.8623 \mathrm{e}-04$ & 4.4236 & $7.5409 \mathrm{e}-05$ & 5.1416 \\
& 16 & $7.0047 \mathrm{e}-08$ & 13.4543 & $1.3512 \mathrm{e}-08$ & 12.4462 \\
& 32 & $1.6426 \mathrm{e}-10$ & 8.7361 & $4.7132 \mathrm{e}-11$ & 8.1633 \\
\hline \multirow{5}{*}{$\alpha=1.5$} & 4 & $1.4409 \mathrm{e}-02$ & & $2.8344 \mathrm{e}-03$ & \\
& 8 & $7.7021 \mathrm{e}-04$ & 4.2255 & $8.2058 \mathrm{e}-05$ & 5.1102 \\
& 16 & $7.3050 \mathrm{e}-08$ & 13.3640 & $1.3431 \mathrm{e}-08$ & 12.5768 \\
& 32 & $2.6752 \mathrm{e}-10$ & 8.0930 & $1.2130 \mathrm{e}-10$ & 6.7908 \\
\hline \multirow{4}{*}{$\alpha=1.8$} & 4 & $1.2195 \mathrm{e}-02$ & & $2.9658 \mathrm{e}-03$ & \\
& 8 & $7.4937 \mathrm{e}-04$ & 4.0244 & $8.7406 \mathrm{e}-05$ & 5.0845 \\
& 16 & $7.4484 \mathrm{e}-08$ & 13.2964 & $1.2990 \mathrm{e}-08$ & 12.7161 \\
& 32 & $1.7250 \mathrm{e}-10$ & 8.7541 & $1.1949 \mathrm{e}-10$ & 6.7643 \\
\hline \multirow{5}{*}{$\alpha=2.0$} & 4 & $1.0871 \mathrm{e}-02$ & & $3.0354 \mathrm{e}-03$ & \\
& 8 & $7.3373 \mathrm{e}-04$ & 3.8891 & $9.0363 \mathrm{e}-05$ & 5.0700 \\
& 16 & $7.4571 \mathrm{e}-08$ & 13.2643 & $1.2546 \mathrm{e}-08$ & 12.8142 \\
& 32 & $4.3006 \mathrm{e}-10$ & 7.4381 & $1.1855 \mathrm{e}-10$ & 6.7255 \\
\hline
\end{tabular}



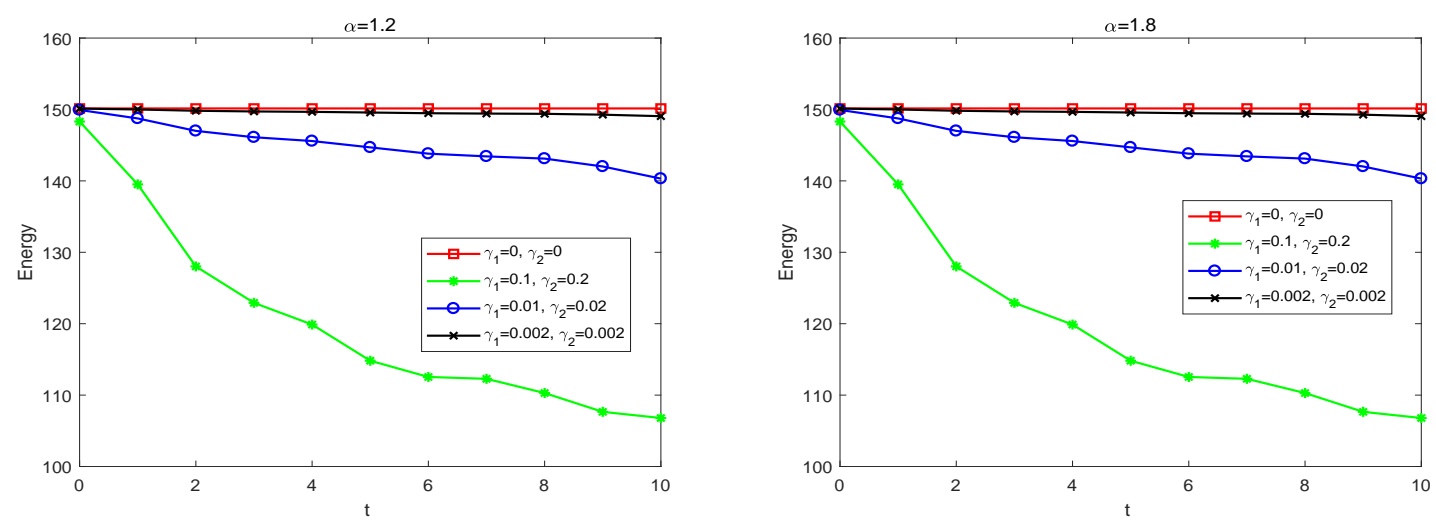

Figure 1: The values of the discrete energy $\mathbf{H}^{n}$ for different $\gamma_{1}$ and $\gamma_{2}$ with time evolution for $\alpha_{1}=1.2$ and $\alpha_{2}=1.8$.

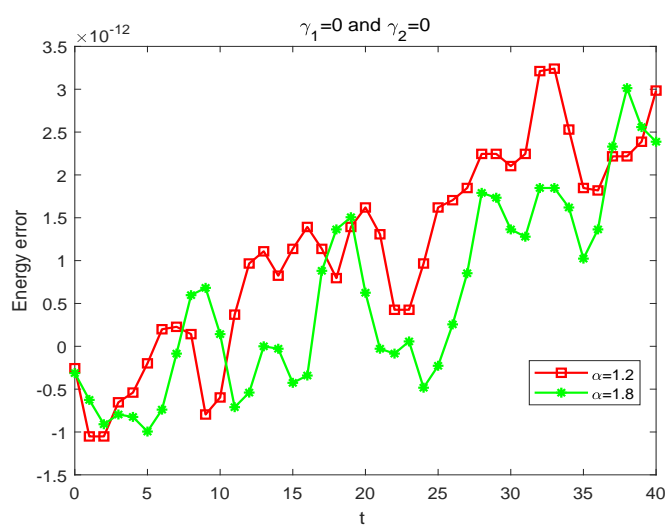

Figure 2: The error of the discrete energy $\mathbf{H}^{n}$ for conservative form,i.e., $\gamma_{1}=0, \gamma_{2}=0$ with time evolution for $\alpha_{1}=1.2$ and $\alpha_{2}=1.8$.

\section{Acknowledgment}

The authors thank the anonymous reviewer for excellent suggestions that helped improve this paper.

\section{References}

\section{References}

[1] A. G. Bratsos, On the numerical solution of the Klein-Gordon equation, Numer. Methods Partial Differential Equations 25 (2009) 939-951.

[2] J. Rashidinia, M. Ghasemi, R. Jalilian, Numerical solution of the nonlinear Klein-Gordon equation, J. Comput. Appl. Math. 233 (2010) 1866-1878.

[3] D. Deng, C. Zhang, Analysis and application of a compact multistep ADI solver for a class of nonlinear viscous wave equations, Appl. Math. Model. 39 (2015) 1033-1049.

[4] A. Campa, T. Dauxois, S. Ruffo, Statistical mechanics and dynamics of solvable models with long-range interactions, Phys. Rep. 480 (2009) 57-159. 

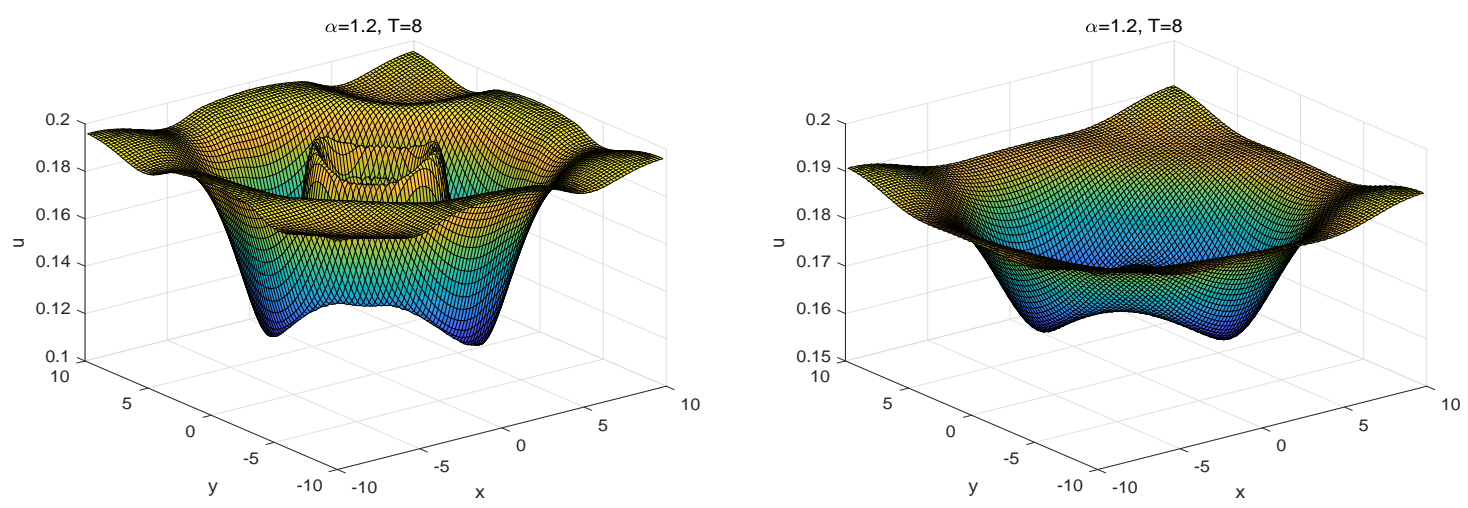

Figure 3: The numerical solution for different $\gamma_{1}$ and $\gamma_{2}$ (Left: $\gamma_{1}=0$ and $\gamma_{2}=0$; Right: $\gamma_{1}=0.5$ and $\gamma_{2}=0$ ) with time evolution for $\alpha_{1}=1.2$ at $T=8$.
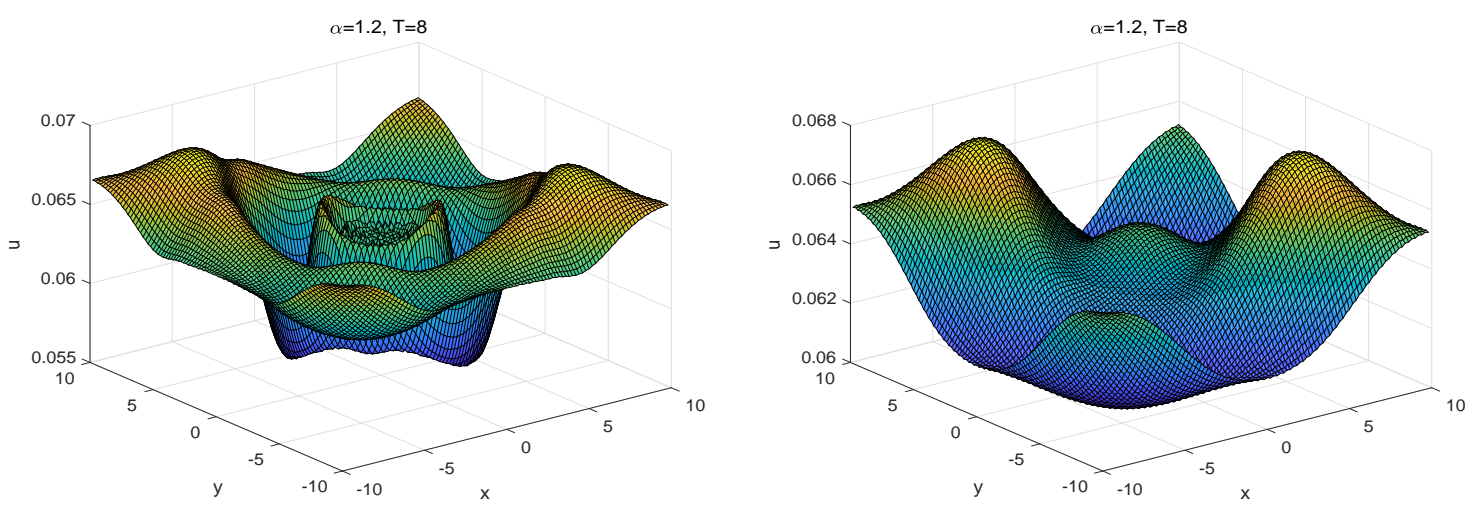

Figure 4: The numerical solution for different $\gamma_{1}$ and $\gamma_{2}$ (Left: $\gamma_{1}=0$ and $\gamma_{2}=0.5$; Right: $\gamma_{1}=0.5$ and $\gamma_{2}=0.5$ ) with time evolution for $\alpha_{1}=1.2$ at $T=8$.

[5] J. L. Shomberg, Well-posedness of semilinear strongly damped wave equations with fractional diffusion operators and $C^{0}$ potentials on arbitrary bounded domains, Rocky Mountain J. Math. 49 (2019) 1307-1334.

[6] S. Chen, X. Jiang, F. Liu, I. Turner, High order unconditionally stable difference schemes for the Riesz spacefractional telegraph equation, J. Comput. Appl. Math. 278 (2015) 119-129.

[7] M. Ran, C. Zhang, Compact difference scheme for a class of fractional-in-space nonlinear damped wave equations in two space dimensions, Comput. Math. Appl. 71 (2016) 1151-1162.

[8] W. Bu, Y. Tang, Y. Wu, J. Yang, Finite difference/finite element method for two-dimensional space and time fractional Bloch-Torrey equations, J. Comput. Phys. 293 (2015) 264-279.

[9] M. Li, X.-M. Gu, C. Huang, M. Fei, G. Zhang, A fast linearized conservative finite element method for the strongly coupled nonlinear fractional Schrödinger equations, J. Comput. Phys. 358 (2018) 256-282.

[10] M. Li, C. Huang, Y. Zhao, Fast conservative numerical algorithm for the coupled fractional Klein-GordonSchrödinger equation, Numer. Algorithms (2019) 1081-1119.

[11] Y. Wang, L. Mei, A conservative spectral galerkin method for the coupled nonlinear space-fractional Schrödinger equations, Int. J. Comput. Math. (2019) 2387-2410.

[12] P. Wang, C. Huang, Structure-preserving numerical methods for the fractional Schrödinger equation, Appl. Numer. Math. 129 (2018) 137-158.

[13] P. Wang, C. Huang, An energy conservative difference scheme for the nonlinear fractional Schrödinger equa- 

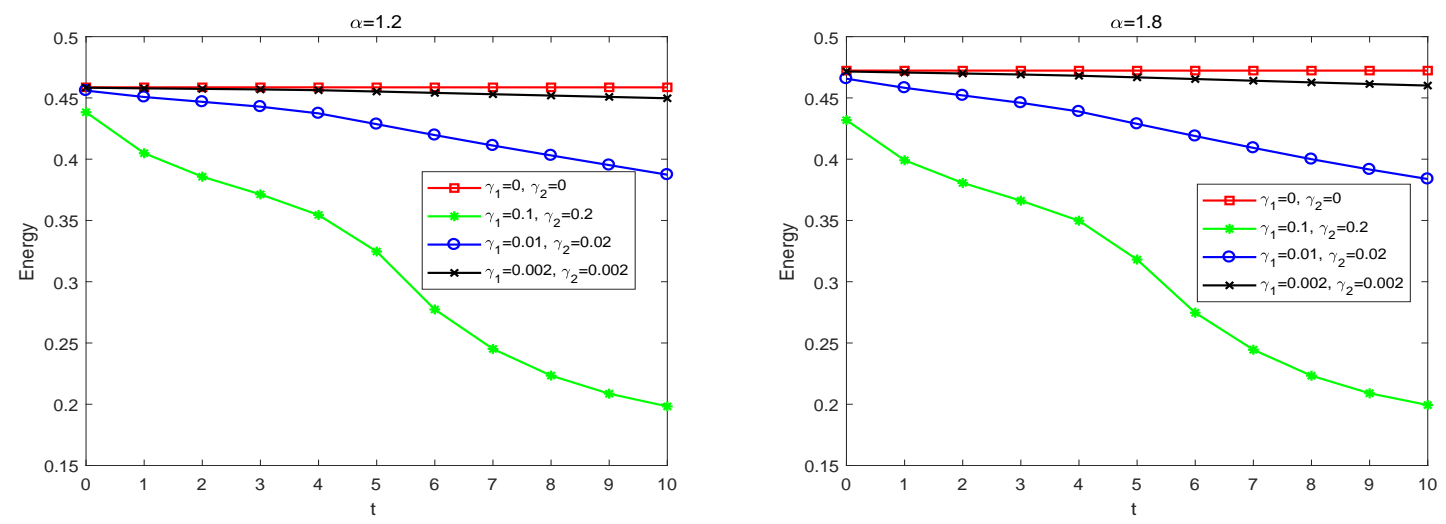

Figure 5: The values of the discrete energy $\mathbf{H}^{n}$ for different $\gamma_{1}$ and $\gamma_{2}$ with time evolution for $\alpha_{1}=1.2$ and $\alpha_{2}=1.8$.

tions, J. Comput. Phys. 293 (2015) 238-251.

[14] J. Wang, A. Xiao, Conservative Fourier spectral method and numerical investigation of space fractional KleinGordon-Schrödinger equations, Appl. Math. Comput. 350 (2019) 348-365.

[15] N. Wang, C. Huang, An efficient split-step quasi-compact finite difference method for the nonlinear fractional Ginzburg-Landau equations, Comput. Math. Appl. 75 (2018) 2223-2242.

[16] H. Zhang, X. Jiang, C. Wang, S. Chen, Crank-Nicolson Fourier spectral methods for the space fractional nonlinear Schrödinger equation and its parameter estimation, Int. J. Comput. Math. 96 (2019) 238-263.

[17] Z. Xing, L. Wen, A conservative difference scheme for the Riesz space-fractional sine-Gordon equation, Adv. Difference Equ. (2018) Paper No. 238, 22.

[18] Y. Fu, W. Cai, Y. Wang, An explicit structure-preserving algorithm for the nonlinear fractional Hamiltonian wave equation, Appl. Math. Lett. 102 (2020) 106-123.

[19] J. E. Macías-Díaz, An explicit dissipation-preserving method for Riesz space-fractional nonlinear wave equations in multiple dimensions, Commun. Nonlinear Sci. Numer. Simul. 59 (2018) 67-87.

[20] J. E. Macías-Díaz, A. S. Hendy, R. H. De Staelen, A pseudo energy-invariant method for relativistic wave equations with Riesz space-fractional derivatives, Comput. Phys. Commun. 224 (2018) 98-107.

[21] J. E. Macías-Díaz, A numerically efficient dissipation-preserving implicit method for a nonlinear multidimensional fractional wave equation, J. Sci. Comput. 77 (2018) 1-26.

[22] J. E. Macías-Díaz, A. S. Hendy, R. H. De Staelen, A compact fourth-order in space energy-preserving method for Riesz space-fractional nonlinear wave equations, Appl. Math. Comput. 325 (2018) 1-14.

[23] J. Xie, Z. Zhang, An effective dissipation-preserving fourth-order difference solver for fractional-in-space nonlinear wave equations, J. Sci. Comput. 79 (2019) 1753-1776.

[24] J. Xie, Z. Zhang, D. Liang, A new fourth-order energy dissipative difference method for high-dimensional nonlinear fractional generalized wave equations, Commun. Nonlinear Sci. Numer. Simul. 78 (2019) 104850, 21.

[25] T. Matsuo, D. Furihata, Dissipative or conservative finite-difference schemes for complex-valued nonlinear partial differential equations, J. Comput. Phys. 171 (2001) 425-447.

[26] X. Yang, J. Zhao, Q. Wang, J. Shen, Numerical approximations for a three-component Cahn-Hilliard phasefield model based on the invariant energy quadratization method, Math. Models Methods Appl. Sci. 27 (2017) 1993-2030.

[27] Y.-W. Li, X. Wu, General local energy-preserving integrators for solving multi-symplectic Hamiltonian PDEs, J. Comput. Phys. 301 (2015) 141-166.

[28] Y. Gong, J. Cai, Y. Wang, Some new structure-preserving algorithms for general multi-symplectic formulations of Hamiltonian PDEs, J. Comput. Phys. 279 (2014) 80-102.

[29] C. Jiang, W. Cai, Y. Wang, A linearly implicit and local energy-preserving scheme for the sine-Gordon equation based on the invariant energy quadratization approach, J. Sci. Comput. 80 (2019) 1629-1655. 
[30] J. Shen, J. Xu, J. Yang, A new class of efficient and robust energy stable schemes for gradient flows, SIAM Rev. 61 (2019) 474-506.

[31] J. Shen, J. Xu, J. Yang, The scalar auxiliary variable (SAV) approach for gradient flows, J. Comput. Phys. 353 (2018) 407-416.

[32] X. Li, J. Shen, Stability and Error estimates of the SAV Fourier-spectral method for the Phase Field Crystal Equation, arXiv (2019).

[33] C. Jiang, Y. Gong, W. Cai, Y. Wang, A Linearly Implicit Structure-Preserving Scheme for the Camassa-Holm Equation Based on Multiple Scalar Auxiliary Variables Approach, J. Sci. Comput. 80 (2020) 1629-1655.

[34] X. Li, J. Shen, H. Rui, Energy stability and convergence of SAV block-centered finite difference method for gradient flows, Math. Comp. 88 (2019) 2047-2068.

[35] F. Zeng, F. Liu, C. Li, K. Burrage, I. Turner, V. Anh, A Crank-Nicolson ADI spectral method for a twodimensional Riesz space fractional nonlinear reaction-diffusion equation, SIAM J. Numer. Anal. 52 (2014) 2599-2622.

[36] N. Wang, M. Fei, C. Huang, G. Zhang, M. Li, Dissipation-preserving Galerkin-Legendre spectral methods for two-dimensional fractional nonlinear wave equations, Comput. Math. Appl. 80 (2020) 617-635.

[37] B. Li, W. Sun, Unconditional convergence and optimal error estimates of a Galerkin-mixed FEM for incompressible miscible flow in porous media, SIAM J. Numer. Anal. 51 (2013) 1959-1977.

[38] D. Li, J. Wang, Unconditionally optimal error analysis of Crank-Nicolson Galerkin FEMs for a strongly nonlinear parabolic system, J. Sci. Comput. 72 (2017) 892-915.

[39] Z. Si, J. Wang, W. Sun, Unconditional stability and error estimates of modified characteristics FEMs for the Navier-Stokes equations, Numer. Math. 134 (2016) 139-161.

[40] H. Zhang, X. Jiang, F. Zeng, G. E. Karniadakis, A stabilized semi-implicit Fourier spectral method for nonlinear space-fractional reaction-diffusion equations, J. Comput. Phys. 405 (2020) 109-141.

[41] M. Ainsworth, Z. Mao, Analysis and approximation of a fractional Cahn-Hilliard equation, SIAM J. Numer. Anal. 55 (2017) 1689-1718.

[42] R. A. Adams, J. F. Fournier, Sobolev spaces, Sobolev Spaces 140 (2003) 713-734.

[43] J. Shen, T. Tang, L. L. Wang, Spectral Methods: Algorithms, Analysis and Applications, 2011. 